\title{
Rapid Eye Movement Sleep Sawtooth Waves Are Associated with Widespread Cortical Activations
}

\author{
Birgit Frauscher, ${ }^{1}$ Nicolás von Ellenrieder, ${ }^{2}$ Irena Dolezalova, ${ }^{1,3}$ Sarah Bouhadoun, ${ }^{1}$ Jean Gotman, ${ }^{2}$ \\ and ${ }^{-}$Laure Peter-Derex ${ }^{1,4,5}$ \\ ${ }^{1}$ Analytical Neurophysiology Lab, Montreal Neurological Institute and Hospital, McGill University, Montreal, Quebec H3A 2B4, Canada, ${ }^{2}$ Montreal \\ Neurological Institute and Hospital, McGill University, Montreal, Quebec H3A 2B4, Canada, ${ }^{3}$ Brno Epilepsy Center, First Department of Neurology, \\ St. Anne's University Hospital and Faculty of Medicine, Masaryk University, Brno, Czech Republic, 65691 Brno, ${ }^{4}$ Center for Sleep Medicine and \\ Respiratory Diseases, Hospices Civils de Lyon, Lyon 1 University, Lyon, F-69000, France, and ${ }^{5}$ Lyon Neuroscience Research Center, Centre National \\ de la Recherche Scientifique, Unité Mixte de Recherche 5292, Institut National de la Santé et de la Recherche Médicale, U1028, Lyon, F-69000, France
}

Sawtooth waves (STW) are bursts of frontocentral slow oscillations recorded in the scalp electroencephalogram (EEG) during rapid eye movement (REM) sleep. Little is known about their cortical generators and functional significance. Stereo-EEG performed for presurgical epilepsy evaluation offers the unique possibility to study neurophysiology in situ in the human brain. We investigated intracranial correlates of scalp-detected STW in 26 patients (14 women) undergoing combined stereo-EEG/ polysomnography. We visually marked STW segments in scalp EEG and selected stereo-EEG channels exhibiting normal activity for intracranial analyses. Channels were grouped in 30 brain regions. The spectral power in each channel and frequency band was computed during STW and non-STW control segments. Ripples $(80-250 \mathrm{~Hz})$ were automatically detected during STW and control segments. The spectral power in the different frequency bands and the ripple rates were then compared between STW and control segments in each brain region. An increase in $2-4 \mathrm{~Hz}$ power during STW segments was found in all brain regions, except the occipital lobe, with large effect sizes in the parietotemporal junction, the lateral and orbital frontal cortex, the anterior insula, and mesiotemporal structures. A widespread increase in high-frequency activity, including ripples, was observed concomitantly, involving the sensorimotor cortex, associative areas, and limbic structures. This distribution showed a high spatiotemporal heterogeneity. Our results suggest that STW are associated with widely distributed, but locally regulated REM sleep slow oscillations. By driving fast activities, STW may orchestrate synchronized reactivations of multifocal activities, allowing tagging of complex representations necessary for REM sleep-dependent memory consolidation.

Key words: gamma; polysomnography; REM; signal analysis; sleep; stereo-EEG

\section{Significance Statement}

Sawtooth waves (STW) present as scalp electroencephalographic (EEG) bursts of slow waves contrasting with the low-voltage fast desynchronized activity of REM sleep. Little is known about their cortical origin and function. Using combined stereoEEG/polysomnography possible only in the human brain during presurgical epilepsy evaluation, we explored the intracranial correlates of STW. We found that a large set of regions in the parietal, frontal, and insular cortices shows increases in $2-4 \mathrm{~Hz}$ power during scalp EEG STW, that STW are associated with a strong and widespread increase in high frequencies, and that these slow and fast activities exhibit a high spatiotemporal heterogeneity. These electrophysiological properties suggest that STW may be involved in cognitive processes during REM sleep.

Received July 11, 2020; revised Sep. 18, 2020; accepted Oct. 6, 2020.

Author contributions: B.F., I.D., and L.P.-D. designed research; B.F., N.v.E., I.D., S.B., and L.P.-D. performed research; B.F., N.v.E., I.D., J.G., and L.P.-D. analyzed data; B.F., N.v.E., I.D., S.B., J.G., and L.P.-D. edited the paper; B.F., N.v.E., J.G., and L.P.-D. wrote the paper; L.P.-D. wrote the first draft of the paper.

This work was supported by the Natural Sciences and Engineering Research Council of Canada RGPIN2020-04127 and RGPAS-2020-00021 to B.F., and the Canadian Institutes of Health Research FDN 143208 to J.G. L.P.-D. was supported by Savoy Epilepsy Foundation Mircea Steriade scholarship. B.F. was supported by Fonds de Recherche du Québec-Santé 2018-2021 Salary Award "Chercheur-boursier clinicien Junior 2." We thank the technicians at the EEG Department of the Montreal Neurological Institute and Hospital, in particular, Lorraine Allard, Nicole Drouin, Chantal Lessard, and Chantal Ménard, as well as Natasha Zazubovits for excellent organization of the MNI SEEG database.

B.F. received speaker's honoraria of UCB Pharma and Eisai. The remaining authors declare no competing financial interests.

Correspondence should be addressed to Birgit Frauscher at birgit.frauscher@mcgill.ca or Laure Peter-Derex at laure.peter-derex@chu-lyon.fr, laure.peter@mail.mcgill.ca.

https://doi.org/10.1523/JNEUROSCI.1586-20.2020

Copyright $\odot 2020$ the authors 


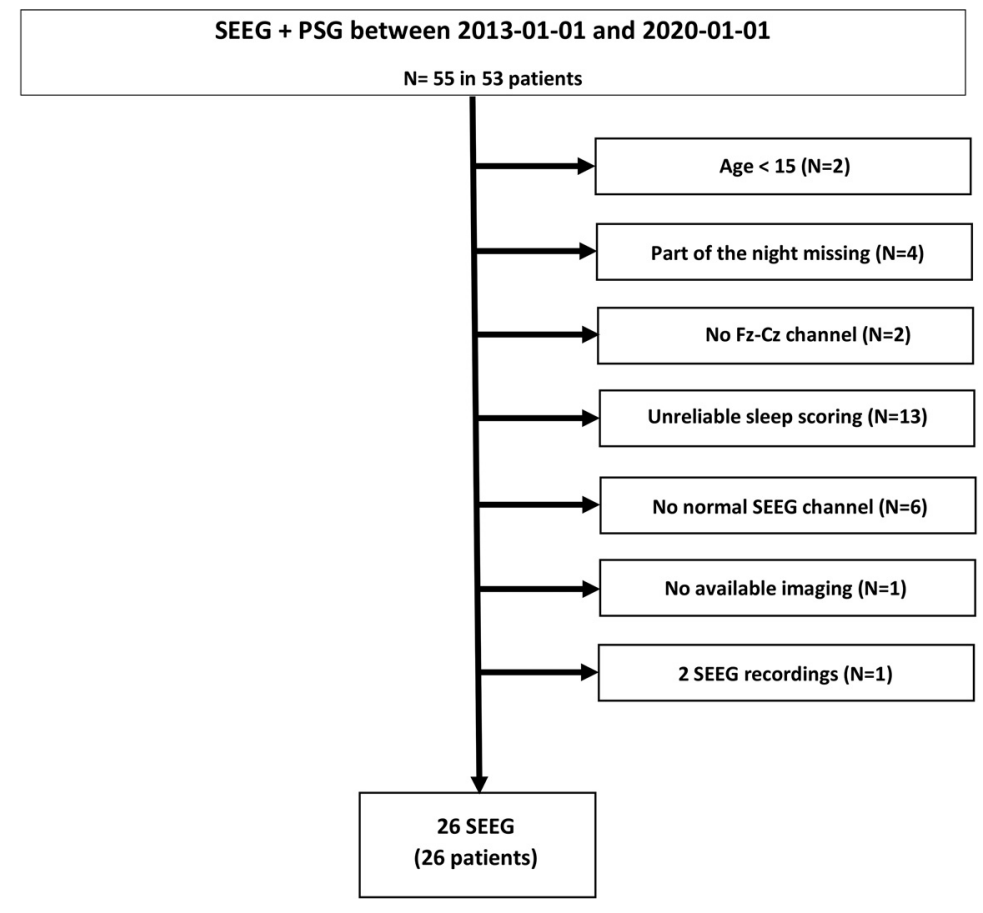

Figure 1. Flowchart of the patient selection process.

\section{Introduction}

Human sleep is characterized by several electrophysiological features resulting from oscillations within cortico-subcortical networks and associated with specific functions (Steriade et al., 1993; Carskadon and Dement, 2017; Luppi and Fort, 2019). Non-rapid eye movement (NREM) sleep rhythms have been well described: they include slow waves, spindles, and ripples, orchestrated in a fine-tuned manner underpinning memory-consolidation processes (Steriade, 2003; Clemens et al., 2007; Diekelmann and Born, 2010; Staresina et al., 2015; Jiang et al., 2019). Paradoxically, whereas rapid eye movement (REM) sleep has been discovered more than 60 years ago, very few studies have focused on sleep oscillations specific to this stage (Dement and Kleitman, 1957; Jouvet and Michel, 1959). Several studies have explored ponto-geniculo-occipital (PGO) waves, providing information about their pontine origin and thalamocortical propagation, including functional neuroimaging and intracranial recording studies in humans (Nelson et al., 1983; Peigneux et al., 2001; Lim et al., 2007; Frauscher et al., 2018a). In contrast, sawtooth waves (STW), which represent another slow oscillation of REM sleep, have been neglected although they have been observed in the scalp electroencephalogram (EEG) since the 1960s (Jouvet et al., 1960; Berger et al., 1962; Rechtschaffen and Kales, 1968).

STW present as bursts of low-frequency (LF) rhythms contrasting with the low-voltage fast desynchronized activity associated with REM sleep (J. Siegel, 2011). Their name refers to their morphology, as their shape resembles notched triangular waves (Berger et al., 1962; Rodenbeck et al., 2006). STW peak in the $\delta$ $(2-3 \mathrm{~Hz})$ frequency and are recorded with the highest amplitude over the vertex of the frontocentral regions (Jouvet et al., 1960; Yasoshima et al., 1984; Broughton and Hasan, 1995). They usually occur after the onset of muscle tone reduction, before a burst of REM, and show an increase in density between the first and last sleep cycles (Geisler et al., 1987; Sato et al., 1997; Pearl et al., 2002). Recently, a high-density EEG (HD-EEG) study identified two potential sources of STW: one frontocentral and one occipitotemporal. The authors also observed a positive in-phase association of STW with low $\gamma$ activity, suggesting that STW may be associated with an "activating" effect (Bernardi et al., 2019). However, no direct cortical recording of STW in different brain areas, including mesial temporal structures, has been reported yet and the functional significance of these REM sleep oscillations remains unknown. No study has investigated the association of STW with ripples in the hippocampus which could underlie some processes related to the memory function of REM sleep (Montgomery et al., 2008; Clemens et al., 2009; Boyce et al., 2017).

Stereo-EEG (SEEG), performed only in drugresistant focal epilepsy patients as part of the presurgical evaluation, allows to record both superficial and deep brain structures in humans with high spatiotemporal resolution. When combined with scalp polysomnography (PSG), SEEG provides the unique opportunity to explore intracranial correlates and regional specificities of sleep oscillations (Nir et al., 2011; Peter-Derex et al., 2012; Frauscher et al., 2018a; Latreille et al., 2020). In the present study, we report the first intracranial study of STW in the human. We aimed: (1) to provide a comprehensive description of intracranial correlates of STW in the $\delta$ frequency band; (2) to measure the level of brain activation during STW, as assessed by the mapping of high-frequency (HF) activity associated with these slow rhythms; and (3) to explore the local spatiotemporal distribution of STW as described for NREM oscillations (Nir et al., 2011; Frauscher et al., 2015). We hypothesize that, given their prominent occurrence before bursts of REM and their frontocentral distribution in the scalp EEG, STW may represent cortical correlates of ascending phasic REM sleep-associated inputs, thus reflecting an activated state involving widespread neocortical regions.

\section{Materials and Methods}

\section{Patient selection}

We reviewed charts of consecutive drug-resistant focal epilepsy patients who had undergone combined intracerebral SEEG recordings and PSG as part of the presurgical epilepsy investigation at the Montreal Neurologic Institute and Hospital between October 2013 and January 2020. Inclusion criteria for the present study were as follows: (1) availability of at least one full night recording with scalp EEG, including at least a scalp $\mathrm{Fz}-\mathrm{Cz}$ derivation + EOG + chin EMG; (2) age over 15 years; (3) available postimplantation imaging for anatomic localization of the individual channels; (4) reliable sleep and STW scoring (i.e., no highly abnormal scalp EEG); (5) presence of at least one SEEG channel with normal activity; and (6) no secondarily generalized tonic-clonic seizures occurring $12 \mathrm{~h}$ before or during the selected night, and no clinical focal seizures during the recording. One patient underwent two SEEG explorations during the study period: the latest recording was used for analysis. The final study sample was comprised of 26 patients (age mean \pm SD, $34 \pm 11.7$ years; 14 women). Figure 1 provides a flowchart for the patient selection criteria. Demographic and clinical characteristics of the study sample are given in Table 1. The study was approved by the Montreal Neurologic Institute and Hospital Review Ethics Board (2014-183).

Intracranial EEG and scalp EEG recordings

An average of 10 (median $=10$, range $7-16)$ depth MNI $(9$ contacts of $0.5-1 \mathrm{~mm}$, distance between contacts $5 \mathrm{~mm}$; 9 patients) or DIXI (10-15 
Table 1. Demographic, neuroimaging, and electrophysiological data of the studied patients ${ }^{\mathrm{a}}$

\begin{tabular}{|c|c|c|c|c|c|c|c|c|}
\hline ID & $\begin{array}{l}\text { Age } \\
\text { (yr) }\end{array}$ & Sex & Epilepsy diagnosis & Seizure-onset zone (SEEG) & MRI & AED (mg/day) & $\begin{array}{l}\text { Channels with physiological activity selected for } \\
\text { STW analysis }\end{array}$ & $\begin{array}{l}\text { No. of } \\
\text { selected } \\
\text { STW }\end{array}$ \\
\hline 1 & 41 & $\mathrm{~F}$ & $\begin{array}{l}\mathrm{R}+\mathrm{L} \text { mesial temporal } \\
\text { lobe epilepsy }\end{array}$ & $\begin{array}{l}\mathrm{R}+\mathrm{L} \text { hippocampus }+ \text { amygdala and } \mathrm{L} \\
\text { fusiform gyrus and heterotopia }\end{array}$ & Periventricular nodular heterotopia & CLO (25), LEV (3000) & $\begin{array}{l}\text { LCa1-7; LCp3-8; LFus4-8; LHp4-8; LOF7-8; } \\
\quad \text { LPc7-8 }\end{array}$ & 81 \\
\hline 2 & 37 & M & $\begin{array}{l}\text { Bilateral temporal lobe } \\
\text { epilepsy }\end{array}$ & $\begin{array}{l}\text { L temporal neocortex }+R \text { mesial } \\
\quad \text { temporal }\end{array}$ & Periventricular nodular heterotopia & CBZ (1400), LEV (3000), CLO (10) & LOF5-10; RCa9-14 & 149 \\
\hline 3 & 37 & M & L temporo-insular epilepsy & $\begin{array}{l}\mathrm{L} \text { temporal mesial and neocortical }+ \\
\text { posterior insula }\end{array}$ & Normal & CLO (30), CBZ (600), TOP (200) & LCi4-5; LOF4-9 & 50 \\
\hline 4 & 57 & $\mathrm{~F}$ & $R$ insular epilepsy & $\mathrm{R}$ insula & Surgical bed (temporal) & LTG (200) Clo (20) & RCa6-9: RCp5-9; Rli7-9; ROF5-9 & 45 \\
\hline 5 & 62 & $\mathrm{~F}$ & $\begin{array}{l}\text { Bilateral mesial temporal } \\
\text { lobe epilepsy }\end{array}$ & $L$ and $R$ hippocampus & Bilateral mesial temporal sclerosis & CBZ (800), CL0 (15), LAC (400) & RA4-9; RH4-9; RHp5-9 & 43 \\
\hline 6 & 41 & M & $\begin{array}{l}\text { Bilateral mesial temporal } \\
\text { lobe epilepsy }\end{array}$ & $\begin{array}{l}\text { L and } R \text { hippocampus }+ \text { amygdala }+ \\
\text { parahippocampus }\end{array}$ & $\mathrm{R}$ mesial temporal sclerosis & CBZ (1400), CLO (10) & RCa1-9; Rla6-9 & 54 \\
\hline 7 & 34 & $\mathrm{~F}$ & $\begin{array}{l}\text { L temporo-occipital } \\
\text { epilepsy }\end{array}$ & $\mathrm{L}$ basal temporo-occipital cortex & Normal & CBZ (1200), CL0 (20), LTG (200) & LA4-7; LCp6-15; LFus7-10; LHp1-5 & 112 \\
\hline 8 & 36 & M & $\begin{array}{l}\text { Bilateral neocortical tem- } \\
\text { poral lobe epilepsy }\end{array}$ & $L+R$ temporal neocortex & L hippocampus atrophy & OXC (1800), LEV (3000), LTG (400) & LFus1-4; RFus1-4 & 65 \\
\hline 9 & 38 & M & $\begin{array}{l}\text { L temporo-occipital } \\
\text { epilepsy }\end{array}$ & $\begin{array}{l}\text { L posterior hippocampus, lingual gyrus } \\
\text { and cuneus }\end{array}$ & L posterior temporal atrophy and gliosis & LEV (3000), PHT (350), Clo (40) & LCp1-8; Lla4-8; L0F1-9 & 39 \\
\hline 10 & 39 & M & $\begin{array}{l}\text { Bilateral temporal lobe } \\
\text { epilepsy }\end{array}$ & $L+R$ temporal mesial and neocortical & R hippocampus atrophy & VPA (2000), Clo (30), LEV (3000) & LCa1-7; LCs1-6; RCs1-3 & 12 \\
\hline 11 & 31 & $\mathrm{~F}$ & $\begin{array}{l}\text { L mesial temporal lobe } \\
\text { epilepsy }\end{array}$ & $\begin{array}{l}\text { L amygdala }+ \text { hippocampus }+ \text { fusiform } \\
\text { gyrus }\end{array}$ & L hippocampus atrophy & LTG (600), CBZ (800) & LCa4-9; LCi1-6; LCp4-9; L0i1-9 & 49 \\
\hline 12 & 27 & $\mathrm{~F}$ & L occipital lobe epilepsy & L lateral temporo-occipital cortex & L lateral occipital FCD & $\operatorname{LAM}(300), \operatorname{CBZ}(400)$ & LCi1-9; LH1-5; L0s4-9; LPc1-9 & 84 \\
\hline 13 & 22 & M & $\begin{array}{l}\text { Bilateral mesial temporal } \\
\text { lobe epilepsy }\end{array}$ & L and $R$ hippocampus + amygdala & Bilateral mesial temporal sclerosis & CBZ (1000), LAM (400), LEV (2500) & LHa5-10; LHp5-12; LOF2-10 & 35 \\
\hline 14 & 30 & M & $\mathrm{L}$ frontal lobe epilepsy & L orbitofrontal (mesial) neocortex & Possible mesial orbitofrontal FCD & $\operatorname{LEV}(1000)$, CLO (20) & LHa6-10; LHp6-10 & 15 \\
\hline 15 & 29 & M & $\begin{array}{l}\text { L mesial temporal lobe } \\
\text { epilepsy }\end{array}$ & L hippocampus + amygdala & $\begin{array}{l}\text { L left mesial temporal sclerosis }+\mathrm{L} \\
\text { frontal FCD }\end{array}$ & CBZ (1200), CLO (20) & LCa1-8; LFP1-10 & 6 \\
\hline 16 & 27 & $\mathrm{~F}$ & L insular lobe epilepsy & L posterior insula & $\begin{array}{l}\text { Posterior Insula / Heschl's gyrus FCD + } \\
\text { surgical bed (temporal neocortex) }\end{array}$ & CBZ (1400), LTG (100), CL0 (40) & LCa1-3; LCp5-8; LM1-5; LS1-4; LSMA1-7 & 16 \\
\hline 17 & 24 & M & $\begin{array}{l}\text { Bilateral temporal mesial } \\
\text { epilepsy }\end{array}$ & L and R hippocampus + amygdala & $\begin{array}{l}\mathrm{R} \text { hippocampus atrophy and } \mathrm{L} \text { hippocam- } \\
\text { pus malformation }\end{array}$ & CLO (15) & LCp5-8; LOF1-10; RCp5-9; RHp7-10; ROF1-10 & 88 \\
\hline 18 & 30 & $\mathrm{~F}$ & $\begin{array}{l}\text { Bilateral temporal mesial } \\
\text { epilepsy }\end{array}$ & $\begin{array}{l}\mathrm{L} \text { and } \mathrm{R} \text { hippocampus + amygdala } \\
\qquad(\mathrm{R}>\mathrm{L})\end{array}$ & Normal & OXC (1800), PHE (125), CL0 (20), TPM (25) & LH5-10; RCa1-6; RCm1-5; RCp1-5; RPc1-6 & 76 \\
\hline 19 & 47 & M & R temporoparietal epilepsy & $\begin{array}{l}\text { R posterior temporal and parietal } \\
\text { neocortex }\end{array}$ & $\begin{array}{l}R \text { postsurgical defect (anterior temporal } \\
\text { resection) }+R \text { ischemic frontal } \\
\text { lesion }\end{array}$ & LEV (4000), LAM (200), CL0 (20), ZNZ (200) & $\mathrm{RCm} 1-3 ;$ RFus 1-4 & 51 \\
\hline 20 & 61 & $\mathrm{~F}$ & $\mathrm{~L}$ temporomesial and basal & $\begin{array}{l}\text { L amygdala, hippocampus and fusiform } \\
\text { gyrus }\end{array}$ & $\begin{array}{l}\text { Ischemic lesion next to } L \text { lateral ventri- } \\
\text { cule and hippocampus atrophia }\end{array}$ & $\begin{array}{l}\text { BRV (100), LAC (150), PER (8), venlafaxine } \\
112.5\end{array}$ & LCa5-10; LF01-9; Lla6-8 and 9-14; LOF9-15 & 59 \\
\hline 21 & 21 & $\mathrm{~F}$ & $\begin{array}{l}\text { R mesial temporal lobe } \\
\text { epilepsy }\end{array}$ & R hippocampus + amygdala & Normal & CLO (5), LAM (500), amitriptyline 75 & LA7-9; LFus1-9; RHp4-10; Rla1-10; Rlp1-15; & 80 \\
\hline 22 & 32 & M & $\begin{array}{l}\text { L fronto-temporo-insular } \\
\text { epilepsy }\end{array}$ & $\mathrm{L}$ insula + temporal $+/-$ frontal & L temporal encephalomalacia & LEV (1500), LAC (200), CL0 (30) & LCm1-5; LCp1-6; LPc1-4 et 8-10; LSMA1-3 & 62 \\
\hline 23 & 26 & $\mathrm{~F}$ & $\begin{array}{l}\text { L mesial and basal tempo- } \\
\text { ral lobe epilepsy }\end{array}$ & $\begin{array}{l}\mathrm{R} \text { hippocampus }+ \text { amygdala }+ \text { fusi- } \\
\text { form gyrus }\end{array}$ & Normal & LAC (200), PER (8), LAC (250), LTG (275) & LCi11-14; LFus4-12; LHp4-11; Lla5-8; LIp3-8 & 51 \\
\hline 24 & 25 & M & $R$ frontal lobe epilepsy & $\begin{array}{l}\text { R posterior orbitofrontal cortex and ante- } \\
\text { rior insula }\end{array}$ & Normal & LAC (150), CL0 (10), Citalopram 10 & $\begin{array}{l}\text { LH5-10; Lla11-14; RFP1-9; LOF8-10; RCa1-7; } \\
\text { RCg1-9; Rla8-15; R0Fa5-9; RSMA1-7 }\end{array}$ & 14 \\
\hline 25 & 26 & $\mathrm{~F}$ & R parieto-occipital epilepsy & $\mathrm{R}$ medial parietal $\mathrm{FCD}$ & $\mathrm{R}$ posterior cingulate $\mathrm{FCD}$ & BRV (100), CBZ (400), LTG (150) & $\begin{array}{l}\text { RA1-8; RCa 1-10; RCg 1-10; RCi 1-10; RCp 1-6; } \\
\text { RDa 8-13; Rla 6-11; Rlp1-6; RFa 1-5; RFp 5- } \\
\text { 10; RSMA 1-5 }\end{array}$ & 64 \\
\hline 26 & 18 & $\mathrm{~F}$ & R occipitotemporal epilepsy & R occipitotemporal cortex & Normal & No treatment (CBZ and PER stopped) & RFus4-9; R0a1-10; R0p1-6 & 52 \\
\hline
\end{tabular}

${ }^{a}$ For anatomic localizations, the abbreviation corresponds to the targeted deepest structures and superficial contacts are located in the lateral neocortex: A, Amygdala (superficial contacts: temporal neocortex, middle temporal gyrus); AED, antiepileptic drugs; a, anterior; BRV, Brivaracetam, CBZ, carbamazepine; $(\mathrm{a}$, anterior cingulate gyrus or medial superior frontal gyrus (superficial contacts: inferior or middle or superior frontal gyrus); $(\mathrm{g}$, cingulate gyrus (superficial contacts: middle frontal gyrus); $\mathrm{Ci}$, isthmus of the cingulate gyrus or precuneus (superficial contacts: angular gyrus); $\mathrm{CL}$, clobazam; $\mathrm{Cm}$, middle cingulate gyrus; $\mathrm{Cp}$, posterior cingulate gyrus or superior parietal lobule or cuneus (superficial contacts: supramarginal gyrus or angular gyrus or postcentral gyrus); (s, superior cingulate gyrus or supplementary motor cortex (superficial contacts: middle frontal gyrus); Da, anterior part of the dysplasia; F, medial frontal (superficial contacts: middle or superior frontal gyrus); Fa, anterior part of the superior or middle frontal gyrus; Fp, posterior part of the superior or middle frontal gyrus; $F$, medial fronto-polar cortex (superficial contacts: superior or middle frontal gyrus); FCD, focal cortical dysplasia; F0, frontal operculum; Fus, fusiform gyrus (superficial contacts: temporal neocortex, inferior or middle temporal gyrus); $\mathrm{H}$, hippocampus; Ha, anterior hippocampus (superficial contacts: temporal neocortex, inferior or middle temporal gyrus); Hp, posterior hippocampus (superficial contacts: temporal neocortex, inferior or middle or superior temporal gyrus); i, inferior: I, insula; la, anterior insula (superficial contacts: frontal operculum); li, inferior insula (superficial contacts: temporal operculum); Ip, insula posterior (superficial contacts: parietal operculum); L, left; LAC, Lacosamide; LEV, levetiracetam; LTG, lamotrigine; M, precentral gyrus; 0a, occipital anterior; 0s, occipital superior (superficial contacts: superior parietal lobule or angular gyrus); OXC, oxcarbazepine; OF, orbitofrontal (deep: medial, superficial: lateral); $0 \mathrm{i}$, occipital inferior, lingual gyrus (superficial contacts: middle temporal gyrus or middle occipital gyrus); Op, occipital posterior, cuneus (superior occipital gyrus), $0 \mathrm{~s}$, occipital superior; $p$, posterior; $\mathrm{P}$, parietal; $\mathrm{Pc}$, parieto-occipital, precuneus (superficial contacts: superior parietal lobule or angular gyrus); PER, perampanel; PHE, phenytoine; PNH, periventricular nodular heterotopia; R, right; s, superior; S, postcentral gyrus; SMA, supplementary motor area (superficial contacts: superior or middle frontal gyrus); TPM, topiramate; VPA, sodium valproate; ZNS, zonisamide. 

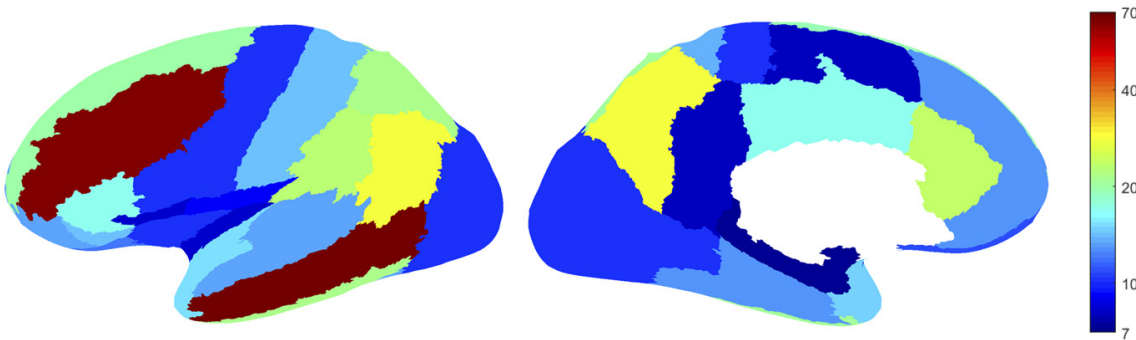

Figure 2. Localization of the channels $(n=544)$ with physiological activity selected for the study. Color of the regions on the inflated cortex represents the number of channels recording from 30 different brain regions. Left, Lateral side; right, medial side. Channels of the left and right hemispheres are pooled together. All regions have $>5$ channels.

contacts of $2 \mathrm{~mm}$, distance between contacts $1.5 \mathrm{~mm}$; 17 patients) electrodes was implanted stereotactically using an image-guided system. The scalp EEG was obtained with subdermal thin wire electrodes (Ives, 2005) at positions $\mathrm{F} 3, \mathrm{~F} 4, \mathrm{Fz}, \mathrm{C} 3, \mathrm{C} 4, \mathrm{Cz}, \mathrm{P} 3, \mathrm{P} 4$, and $\mathrm{Pz}$ (except for 1 patient who only had $\mathrm{Fz}, \mathrm{Cz}$, and $\mathrm{Pz}$ and another one with all but the $\mathrm{F} 3$ electrode). In the night of the sleep recording, which was at least $72 \mathrm{~h}$ after implantation (median 6d, range 3-19), additional electrodes for EOG and chin EMG were applied. The EEG signal was high-pass-filtered at $0.1 \mathrm{~Hz}$, low-pass-filtered at 500 or $600 \mathrm{~Hz}$ (depending on the EEG system), and sampled at $2000 \mathrm{~Hz}$. EEGs were recorded using the Harmonie EEG system (Stellate) from 2010 to 2017 and the Neuroworkbench EEG system (Nihon Kohden) after 2017. Recordings were acquired with a common reference (epidural electrode fixed in the bone, far from the epileptic field). For sleep scoring and scalp analysis, we used a bipolar montage for the scalp EEG (F3-C3; C3P3; Fz-Cz; Cz-Pz; F4-C4; C4-P4). We did not use a traditional mastoid reference montage because of the proximity of some depth electrodes to the mastoid region and the risk of infection, as well as the potential contamination of such a reference by epileptic activity in case of a temporal epileptic focus. All intracranial analyses were done using bipolar montages with the neighboring contacts on the SEEG electrode in the depth EEG.

\section{Selection and localization of intracranial channels recording physiologi-} cal activity

In order to select channels representing as closely as possible normal brain activity, we followed a procedure identical to that of our earlier studies (Frauscher et al., 2018c; von Ellenrieder et al., 2020). In each patient, channels located within the seizure-onset zone and/or the lesion in case of MRI abnormality were excluded. Among the remaining channels, only those exhibiting no or very rare epileptic spikes and no slow-wave anomaly were selected for STW analyses. Thus, a total of 544 bipolar channels (median 19 per patient, range 5-67) were selected. The anatomic localization of electrodes was determined by coregistering preimplantation MRI and postimplantation CT or MRI of each subject, and individual coordinates were then standardized in a common stereotaxic space using minctools (http://bic-mni.github.io/) and the Intraoperative Brain Imaging System framework as previously described (Drouin et al., 2017; Frauscher et al., 2018c). We grouped the channels recording from the same brain region according to a brain segmentation template (Landman and Warfield, 2012). The original template partitions the cerebral brain matter in 60 regions per hemisphere. We joined the channels from both hemispheres, and joined neighboring regions of the template (in the same lobe) until $>5$ channels were included in each brain region following what we did in our previous works (Frauscher et al., 2018c; Latreille et al., 2020). This led to a template with 30 brain regions: 29 neocortical regions and the mesiotemporal region. Figure 2 shows the template and the number of channels recording from each brain region.

\section{Signal analysis}

Sleep and STW scoring. Sleep scoring was manually performed in $30 \mathrm{~s}$ epochs in the scalp EEG, blind to SEEG data, according to American Academy of Sleep Medicine criteria (Berry et al., 2017) by board-certified neurophysiologists (B.F. or L.P.-D.). STW were manually double-marked on scalp EEG independently by two neurophysiologists (I.D. and L.P.-D.) during all REM sleep episodes. They were defined as bursts of consecutive surface-positive $2-5 \mathrm{~Hz}$ frontocentral bilateral synchronous symmetric waves with an amplitude of $20-100 \mu \mathrm{V}$, an angle $>80^{\circ}$, and with a slow incline to a negative peak with a following steep linear decline ending in a positive peak (Sato et al., 1997). We increased the duration criteria from " $\geq 3$ consecutive waves" to " $\geq 2 \mathrm{~s}$ duration burst of consecutive waves" to allow us a wider frequency range for spectral analysis, but also to improve specificity of our detection (for examples, see Figs. 3, 4). The overlap (lasting at least $2 \mathrm{~s}$ ) of the markings obtained from the two raters was selected for further analysis. When events were marked as two segments by one scorer and as single one by the other scorer, the first of the two overlaps was selected. In order to characterize the occurrence of STW with respect to tonic/phasic REM sleep and to choose adequate matched control segments for detection of intracranial correlates of STW, bursts of REM were marked on the EOG derivation, without information about STW marking (S.B.) when they fulfilled the following definition: bursts $\geq 3 \mathrm{~s}$ of sharp onset eye movements clearly standing out the background EOG (Campana et al., 2017). Segments of REM sleep containing bursts of REM were considered as phasic REM sleep. Both scalp and intracranial STW (iSTW) analyses were performed by comparing the STW segments marked on scalp EEG with control segments of $2 \mathrm{~s}$ duration chosen close in time but outside STW markers from both scorers. Each control segment was derived as a weighted average of control variables associated to a $2 \mathrm{~s} \mathrm{segment}$ in tonic REM sleep and another $2 \mathrm{~s}$ segment in phasic REM sleep (both preceding the considered STW segment but outside it and with a $2 \mathrm{~s}$ shift between STW segment onset and control segment offset), with the weights chosen as to respect the proportion of tonic and phasic sleep present in the STW segment. The choice not to select control segments immediately preceding STW segments was made on the observation of asynchrony between scalp STW and $\delta$ bursts recorded in intracranial channels, with possible onset of these bursts slightly before scalp STW onset (for an example, see Fig. $3 A$ ). The $2 \mathrm{~s}$ shift thus allowed us to be sure not to include the beginning of the bursts in the control segment for intracranial analyses. This control segment is illustrated in Figure 4.

Scalp STW analysis. The power spectral density (PSD) of each STW and control segment was estimated with the Welch method (Hamming window, 1 s duration, $50 \%$ overlap). A per-channel PSD was computed averaging all the STW or control segments from that channel. A grand average of all channels (either $\mathrm{Fz}-\mathrm{Cz}$ or $\mathrm{Cz}-\mathrm{Pz}$ ) across patients was also computed.

iSTW analysis. To analyze the intracerebral correlates of scalp STW, we computed the power during each STW and control segment in different frequency bands $(0.3-2-4-8-13-16-20-30-59,61-80-$ $119,121-239 \mathrm{~Hz}$, sixth-order pass-band elliptical IIR filters with $0.25 \mathrm{~dB}$ ripple in the passband and $20 \mathrm{~dB}$ attenuation in the rejection band, applied in both directions to achieve a zero-phase filtering with $40 \mathrm{~dB}$ attenuation). The power of each segment and channel was log-transformed. Next, the distribution of the power in each band was obtained for all control segments in each channel of each patient. The $z$ score of each STW segment was computed based on the distribution of corresponding control segments in that channel. The mean $z$ score of all STW segments in a channel was used to characterize the behavior of the channel of this patient during the scalp STW. The average of mean $z$ scores from all channels recording from the same brain region across patients was computed for each brain region, at different spatial scales (lobar and sublobar as defined using the 30 regions of the segmentation template).

In order to visualize the precise evolution in time of the changes in different intracranial EEG frequency bands at the time of scalp detected STW, the time-frequency analysis of each STW and control segment was performed with a short time Fourier transform (Hamming window, $1 \mathrm{~s}$ duration, $50 \mathrm{~ms}$ step). The time-frequency maps show the average change in spectral power during all STW segments in a channel with respect to the time-averaged maps of the control segments in that channel. Average maps of channels recording from the same brain region were also created for each region. 


\section{A $\quad$ F3-C3 $^{-C_{3}}$ \\ C3-P3 \\ $\mathrm{Fz}-\mathrm{Cz}$ \\ $\mathrm{Cz}-\mathrm{Pz}$ \\ F4-C4 \\ C4-P4 \\ EOG \\ EMG \\ Supplementary motor cortex \\ Middle cingulate gyrus \\ Pre-cuneus}
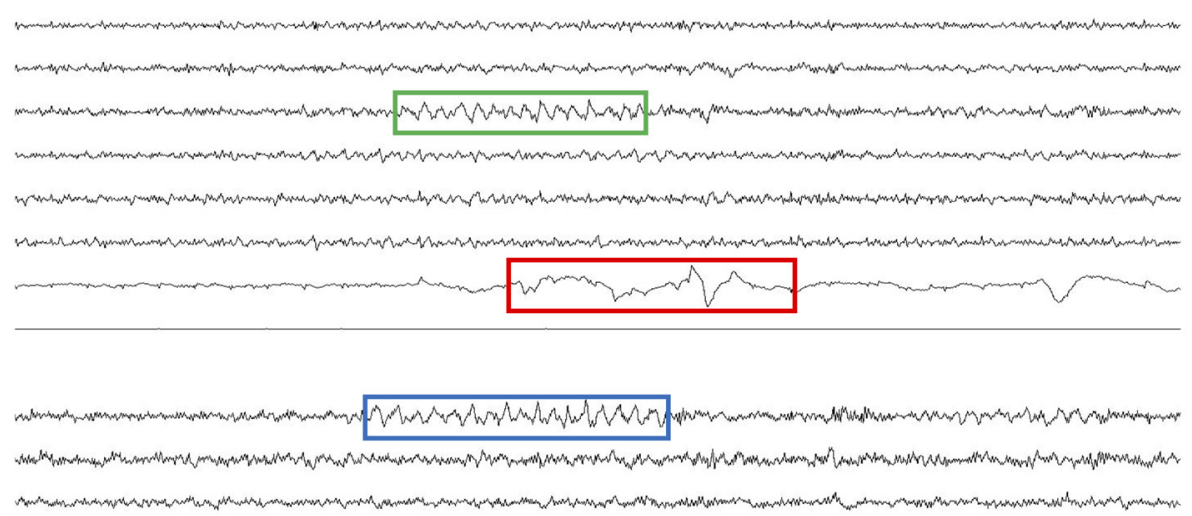

Post-central gyrus Opercular part of the inferior frontal gyrus Posterior insular cortex
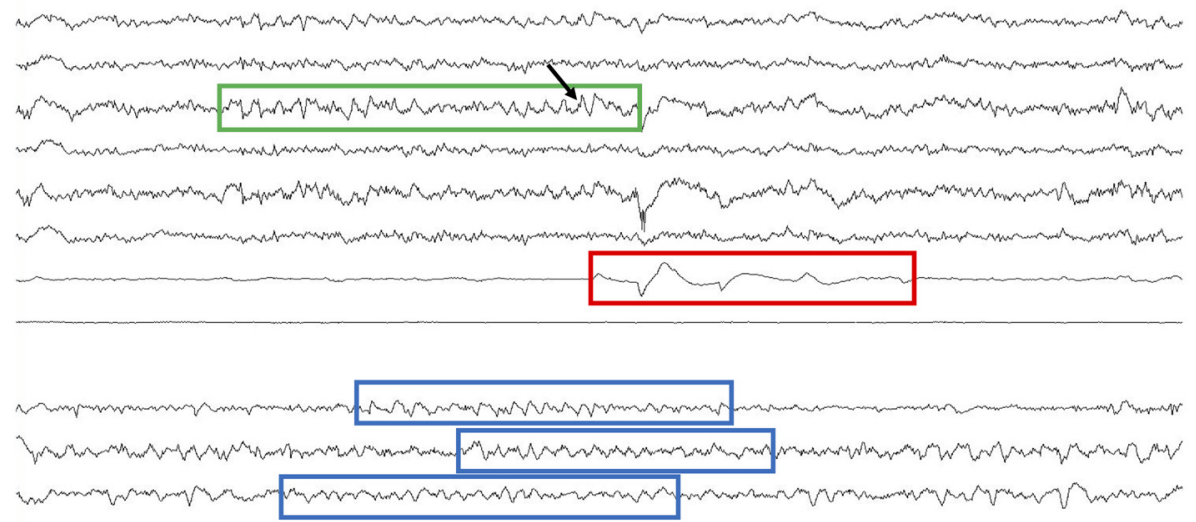

Figure 3. Example of scalp EEG and iSTW. A, Local iSTW (Patient 22). Scalp STW are observed in the frontocentral vertex (green box) before a burst of REM (red box). A burst of isTW (blue box) is observed in the supplementary motor cortex. Note the very focal aspect of the iSTW burst, which is not observed in other intracranial channels. B, Diffuse asynchronous STW (Patient 25). Scalp STW are observed in the frontocentral vertex (green box) before a burst of REM (red box). See the characteristic notch (black arrow). Bursts of iSTW are recorded in three intracranial regions (blue boxes). Note the asynchrony in the onset and offset of iSTW bursts between brain areas.

The occurrence of ripples (oscillations in the $80-250 \mathrm{~Hz}$ band) during STW and control segments was studied with a published HF oscillation detector (Frauscher et al., 2018b). The total number of ripples and total duration of STW/control segments was used to compute the ripple rate in each channel and condition. The median rate among all channels recording from the same brain region was computed to quantify the behavior of each region during STW and control conditions. We also computed the average $z$ score of the power change for segments with and without ripples, to study the association between the change in power and the occurrence of ripples.

The variability of the STW segments was studied with the same $z$ scores described previously. In a particular patient, the correlation coefficient between the $z$ scores in different channels/frequency bands gives an idea of the spatial and frequency variability. When considering all patients, an unsupervised clustering was conducted to group the different channels, using the mean $z$ scores in different bands as features. The clustering was done by minimizing the within group inconsistency coefficients of the links of a hierarchical cluster tree built using the Ward distance (Jain and Dubes, 1988).

\section{Statistics}

We used one-sample two-sided $t$ tests to determine whether $z$ scores from different brain regions, frequency bands, and/or conditions (with or without ripples) were statistically different from zero. We adopted Dunnett's correction for multiple comparisons. Since statistical significance does not imply practical relevance, we also reported the effect size observed for all the comparisons we made. The reported effect size measure is Cohen's $d$.

\section{Results}

\section{Scalp STW}

From 1881 marks from Scorer 1 and 1723 from Scorer 2, 1452 common segments lasting at least $2 \mathrm{~s}$ were selected (median 51.5 per patient, range 6-149; for examples of STW, see Figs. 3, 4). The median duration of the marked STW bursts (overlap of both scorers) was $3.3 \mathrm{~s}$ (Scorer 1: $3.7 \mathrm{~s}$; Scorer 2: $3.6 \mathrm{~s}$ ) and the median delay between both scorers' markings was $156 \mathrm{~ms}$ for the STW onset and $260 \mathrm{~ms}$ for the STW offset.

\section{Rate and occurrence of STW in relation with phasic/tonic REM sleep}

The median STW rate among patients was 0.66 STW per minute (range $0.20-1.44$ ). STW bursts represented $4.1 \%$ of the total REM sleep duration. However, a threefold increase in STW density was observed at the time of the onset of bursts of REM. This increase was noticeable from $-1 \mathrm{~s}$ to $4 \mathrm{~s}$ after the onset of the burst of REMs (Fig. 5A). The proportion of tonic/phasic REM sleep during the whole REM sleep duration ( $36.9 \mathrm{~h}$ ) in the 26 patients was $75 \% / 25 \%$. STW onset followed this distribution (76\% during tonic REM sleep/24\% during phasic REM sleep), whereas STW offset was more prevalent in phasic REM sleep than expected (56\% during tonic REM sleep/44\% during phasic REM sleep), thus reflecting the fact that STW occurred at the tonic/phasic REM transition. Among STW that started during phasic REM sleep, $80 \%$ also ended in phasic REM sleep.

\section{Intra-STW frequency}

Spectral analysis of STW segments showed a peak between 2 and $4 \mathrm{~Hz}$ (median $2.7 \mathrm{~Hz}$ ), with higher average power over the frontocentral leads compared with centroparietal leads (Fig. 5B). The average increase in spectral power compared 
(a)

(b)

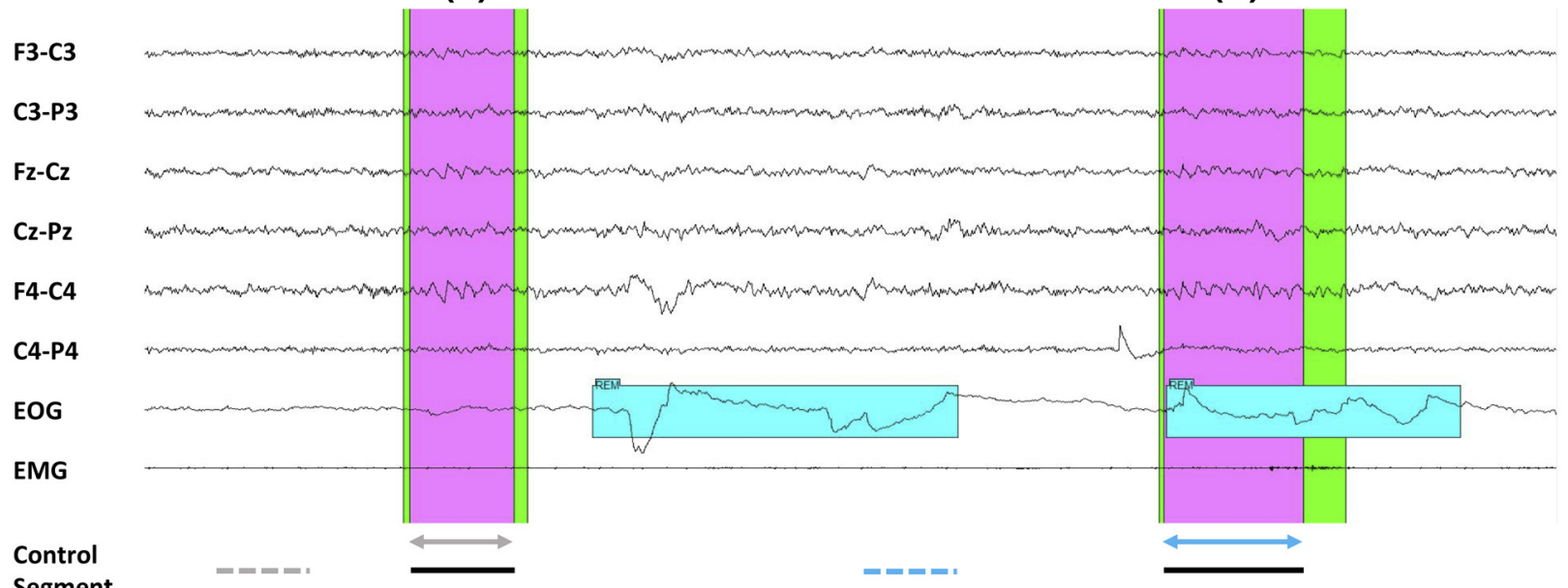

(c)

F3-C3

C3-P3

Fz-Cz

$\mathrm{Cz}-\mathrm{Pz}$

F4-C4

C4-P4

EOG

EMG

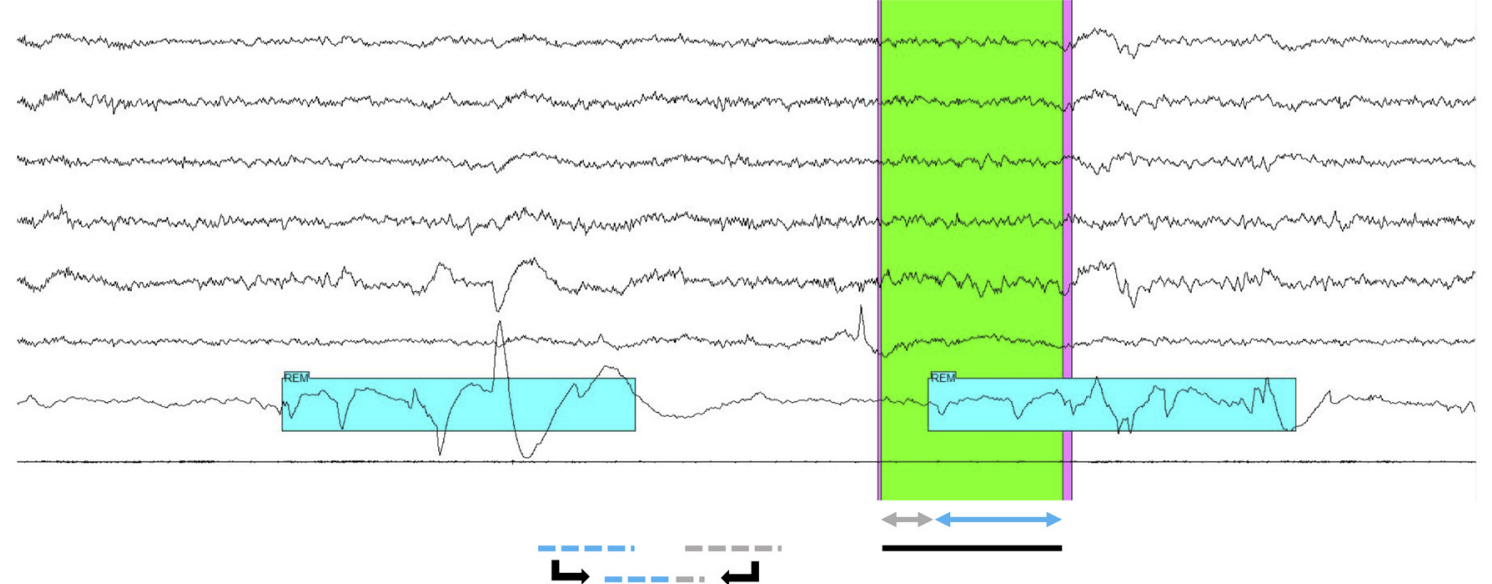

Control

Segment

Figure 4. Double scoring of STW and choice of control segment (example Patient 7). Both scalp and iSTW analyses were performed by comparing the overlap of periods corresponding to STW marked on scalp EEG (green marker represents Scorer 1; pink marker represents Scorer 2; black line below indicates the overlap) with a control segment chosen outside any STW marker. The control segment was derived from a $2 \mathrm{~s}$ segment in tonic and another $2 \mathrm{~s}$ segment in phasic REM sleep (both as close as possible to the STW segment but separated from $2 \mathrm{~s}$ ), with the weights chosen as to respect the proportion of tonic and phasic REM sleep as in the STW segment. Example (a), only tonic (gray arrow) STW segment. In this case, the control segment is selected in tonic (dotted gray) REM sleep; example (b), only phasic (blue arrow) STW segment; the control segment is selected in phasic (dotted blue) REM sleep; example (c), mixed tonic and phasic STW segment. The equivalent segment is a $2 \mathrm{~s}$ segment built with parts of $2 \mathrm{~s}$ phasic and tonic segments.

with the control segments also peaked between 2 and $4 \mathrm{~Hz}$ (median $3.2 \mathrm{~Hz}$ ) (Fig. 5C).

\section{iSTW}

Given the median intra-STW frequency observed in the scalp EEG, we will refer to iSTW to describe the increase in the $2-4 \mathrm{~Hz}$ frequency band at the time of scalp-detected STW compared with control segments.

Brain areas involved in iSTW represent a parieto-fronto-insular network

When looking at a lobar level, iSTW were found in all but the occipital lobe, with a significant increase in the $2-4 \mathrm{~Hz}$ frequency band in the fronto-parieto-temporal cortex and particularly large effect size in the insular cortex (Fig. 6A). More precisely, several parietal areas were involved, including the angular gyrus (Cohen's $d=0.97)$, superior parietal cortex $(d=1.14)$, and posterior cingulate gyrus $(d=1.30)$. A large effect size was also found in the anterior $(d=1.04)$ and the posterior insula $(d=1.84)$. Regarding the frontal lobe, the frontal operculum $(d=1.12)$, the inferior frontal gyrus $(d=0.94)$, and the medial orbitofrontal cortex $(d=1.12)$ exhibited strong $2-4 \mathrm{~Hz}$ increases, and a weaker increase was also observed in the superior frontal gyrus $(d=$ $0.53)$. In the temporal lobe, iSTW were found in the mesial temporal structures $(d=1.08)$ and to a lower extent in the superior $(d=0.76)$ and inferior $(d=0.54)$ temporal gyrus (Fig. 6B,C). However, in most of these regions, the $2-4 \mathrm{~Hz}$ increases did not remain significant after correction for multiple comparisons. No significant increase in $\delta$ band power or large effect size was observed when analyzing the pre-STW segment, arguing against a systematic pattern of propagation of iSTW.

Many regions exhibit HF activity during STW

Interestingly, a concomitant significant increase in HF activity (high $\beta$ and mostly $\gamma[30-80 \mathrm{~Hz}]$ and ripple band $[80-240 \mathrm{~Hz}]$ ) was observed in all lobes, except the occipital lobe where an increase in $\theta$ to low $\beta$ activity was found (Fig. 6A). More specifically, almost all regions in which iSTW were observed also exhibited an increase in $\gamma$ and ripple band activity, significant in the angular gyrus and the superior parietal cortex. Additionally, isolated HF activity was found in many other regions, including the precuneus, the supramarginal gyrus and the parietal operculum, 
A

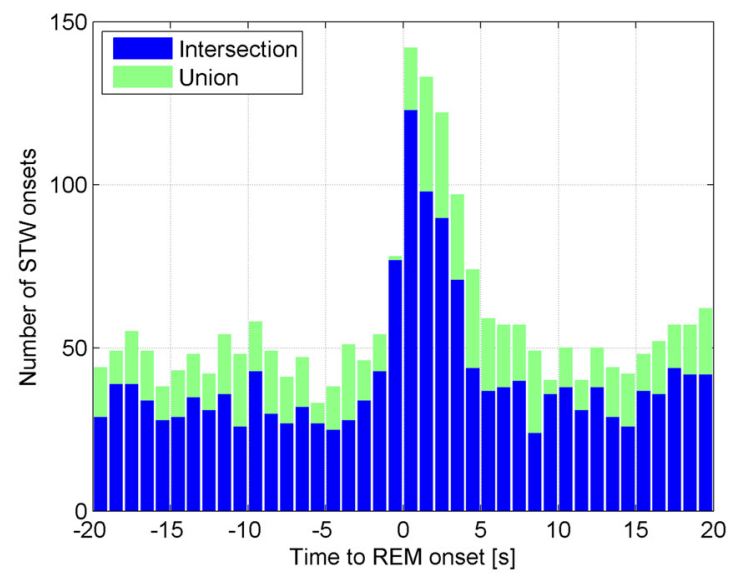

B

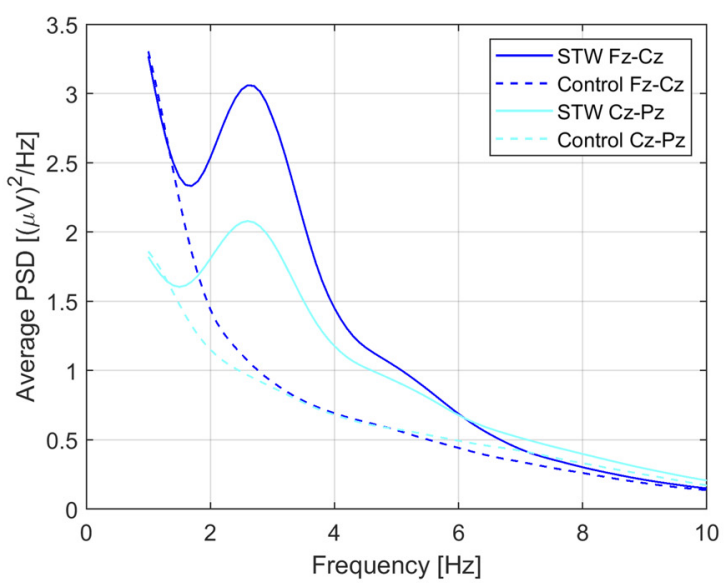

C

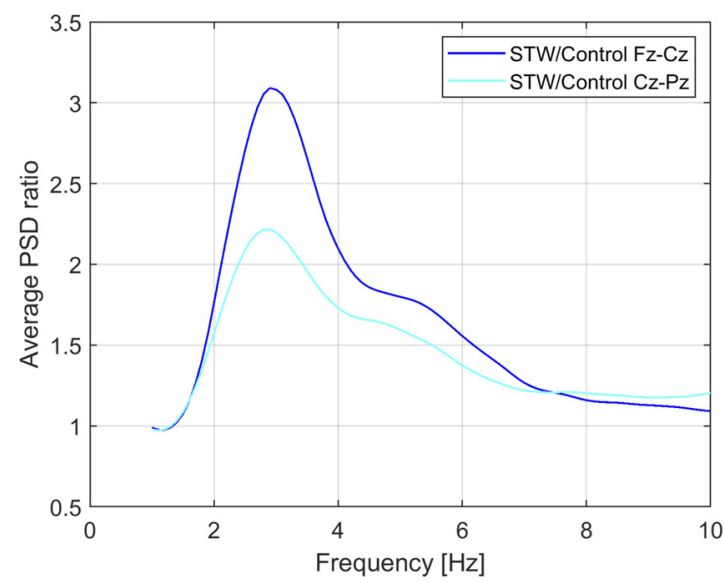

Figure 5. Characteristics of scalp EEG STW. $\boldsymbol{A}$, Distribution of STW onset with respect to the onset of REM bursts. Blue represents intersection (overlap) of STW segments marked by the 2 scorers $(n=1452)$. Green represents union of STW segments marked by the 2 scorers $(n=2068$ ). A large increase is observed around time 0 (from $-1 \mathrm{~s}$ to 4 [intersection] or $5 \mathrm{~s}$ [union]), indicating that STW are more common at the beginning of REM bursts. $\boldsymbol{B}$, Average of log-transformed PSD of STW segments calculated in $\mathrm{Fz}-\mathrm{Cz}$ and $\mathrm{Cz}-\mathrm{Pz}$ derivation when available $(N=24)$, and of control segments (preSTW control and equivalent tonic/phasic control). A peak at $2.7 \mathrm{~Hz}$ is observed only for STW segments, of higher amplitude in frontocentral than in centroparietal derivations. C, Ratio of PSD between STW segments and control segments showing a PSD increase in the 2-4 Hz frequency range (peak at $3.2 \mathrm{~Hz}$ ) during STW, of higher amplitude in frontocentral than in centroparietal derivations. the middle frontal gyrus, the medial frontal cortex, the anterior and middle cingulate cortex, the middle and superior temporal gyrus, the temporal pole, as well as the fusiform gyrus and the parahippocampal gyrus. A very strong effect size was observed in the precentral gyrus, the supplementary motor cortex, and in mesial temporal structures, although this increase in $30-240 \mathrm{~Hz}$ power did not remain significant after correction for multiple comparisons (Fig. $6 B, C$ ). At the same time, several areas in the frontal and in the parietal lobe exhibited a decrease in $\alpha-\beta$ frequencies, significant in the precentral gyrus. Time-frequency analysis of peri-STW segments confirmed these findings, showing that the increase in $\gamma$ and ripple band activity and the decrease in $\alpha-\beta$ activity occurred at the time of iSTW and of scalp STW bursts (Fig. 7).

In order to explore more precisely HF activity, the ripple rate was estimated in STW and control segments. Large variations were observed within regions, but several areas had a median ripple rate $>0.2 / \mathrm{min}$ (Fig. 8). This cutoff was used as it was of the same order as the false detection rate of the automatic detector (Frauscher et al., $2018 b)$. Median ripple rates in these regions were, from the highest to the lowest: mesial temporal region $(0.42 / \mathrm{min})$, posterior cingulate cortex $(0.41 / \mathrm{min})$, occipital lobe $(0.41 / \mathrm{min})$, orbitofrontal cortex and frontal pole $(0.38 / \mathrm{min})$, precuneus $(0.28 / \mathrm{min})$, angular gyrus $(0.23 / \mathrm{min})$, and planum temporale, superior temporal gyrus, and transverse temporal gyrus $(0.20 / \mathrm{min})$. During control segments, only the occipital lobe showed a ripple rate $>0.2 / \mathrm{min}(1.19 / \mathrm{min})$. Interestingly, the comparison of spectral power between STW segments associated and not associated with ripples showed a significant increase not only in the low ripple band $(80-120 \mathrm{~Hz})$ $(p<0.0001$, Cohen's $d=0.64)$ but also in the $2-4 \mathrm{~Hz} \delta$ band $(p<0.0001$, Cohen's $d=0.30)$, suggesting an association between high amplitude iSTW and the presence of ripples.

As shown above, scalp EEG STW occurred mostly within the vicinity of bursts of REM. In order to assess specifically whether changes in intracranial activity during STW were related to the underlying phasic or tonic microstate, we compared directly the 432 "pure tonic STW" segments (i.e. separated by at least $12 \mathrm{~s}$ from the preceding burst of REMs: to allow for a $2 \mathrm{~s}$ control segment separated by $2 \mathrm{~s}$ from the STW segment onset) and by at least $8 \mathrm{~s}$ from the following burst of REM) with the 342 "pure phasic STW" segments (with start and end during bursts of REM as well as the control segment). This $8 \mathrm{~s}$ time window was chosen in line with previous research (De Carli et al., 2016; Simor et al., 2019). No significant difference was found between "pure tonic" and "pure phasic" STW, except for an increase in the $6-8 \mathrm{~Hz}$ band in the medial orbital gyrus and in the $120-140 \mathrm{~Hz}$ band in the middle cingulate cortex during "pure tonic" STW segments.

\section{Spatiotemporal heterogeneity: STW as local phenomena}

Finally, we investigated the variability in the involvement of the explored brain areas during iSTW. From a temporal point of view, in a given region, a high heterogeneity was observed from one scalp-detected STW segment to another. From a spatial point of view, the set of regions showing iSTW was also highly variable and asynchrony between iSTW could be observed (Fig. 3B). This variability was not only found at the regional level, as described above, but was also observed within regions between channels and involved high $\delta$ band as well as higher-frequency bands.

An example of temporal and spatial variability is illustrated in Figure 9 (Patient 18). It shows that the $z$ score of the power increase in the $2-4 \mathrm{~Hz}$ band and in the low ripple band (80$120 \mathrm{~Hz}$ ) for the different STW events in a given channel exhibits a high temporal variability. The time-series of the $z$ score of the 
A

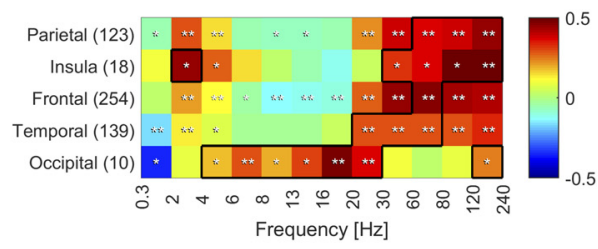

C
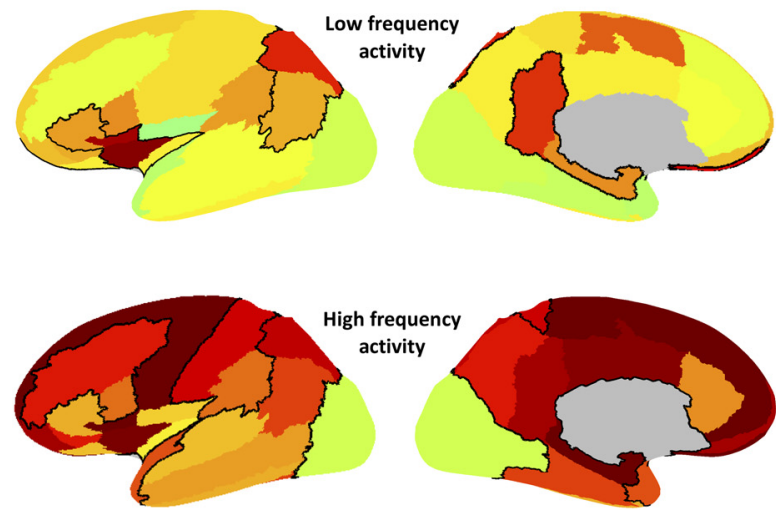

B

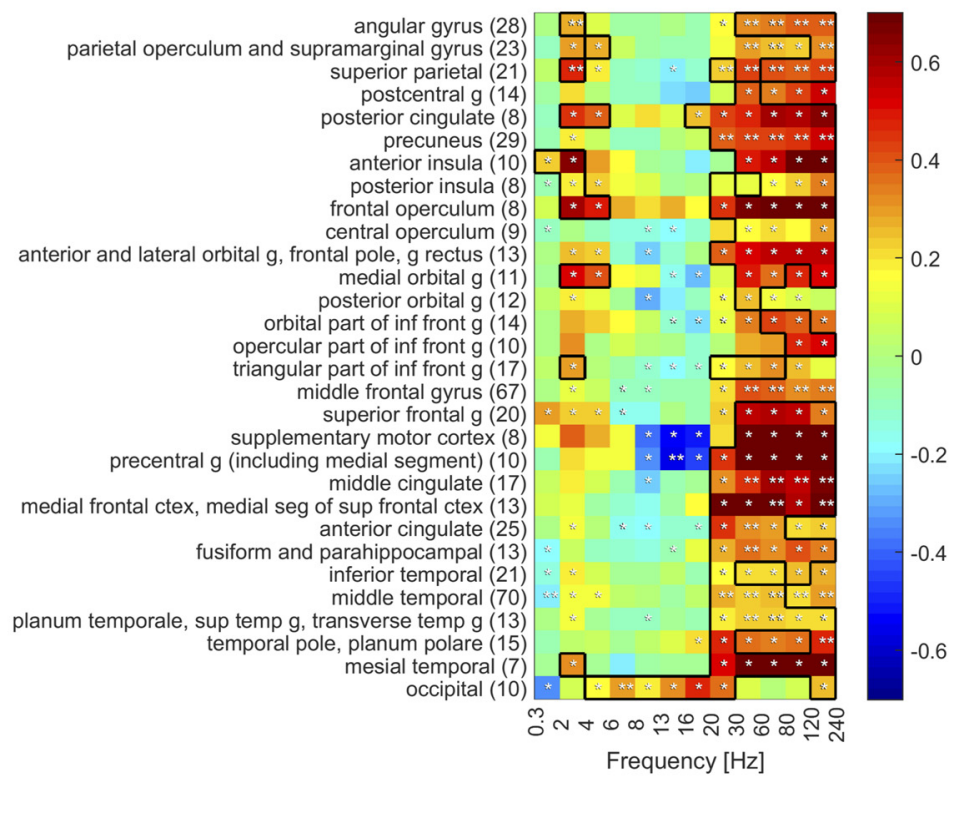

Figure 6. Spectral changes in SEEG signal during scalp-detected STW according to brain area. Color represents the average $z$ score of all channels in the region with respect to the control segments distribution. Black outline indicates a large effect size (Cohen's $d>0.8)$. $\boldsymbol{A}$, Lobar scale. $\boldsymbol{B}$, Sublobar scale. A significant increase in $2-4 \mathrm{~Hz}$ power during STW segments is observed in all lobes but the occipital lobe, with large effect size in the insular cortex. At the same time, a strong and significant increase in HF activity is observed in the same regions. When looking at the sublobar scale, we see that posterior parietal areas, including the parietotemporal junction, inferior frontal region, and anterior insula, are particularly involved in this $\delta$ activity. The increase in HF activity involves almost all areas, except for the occipital cortex. Average $z$ score significantly different from zero: *uncorrected; **corrected). The number of channels averaged in each brain region is indicated in parentheses. C, Representation on the inflated cortex of the variation in LF (2-4 Hz, top) and HF (30-240 Hz, bottom) activity during scalp-detected STW with respect to control segments. Left, Lateral side; right, medial side. The channels of the left and right hemispheres are pooled together. Abbreviations: $g$, gyrus.
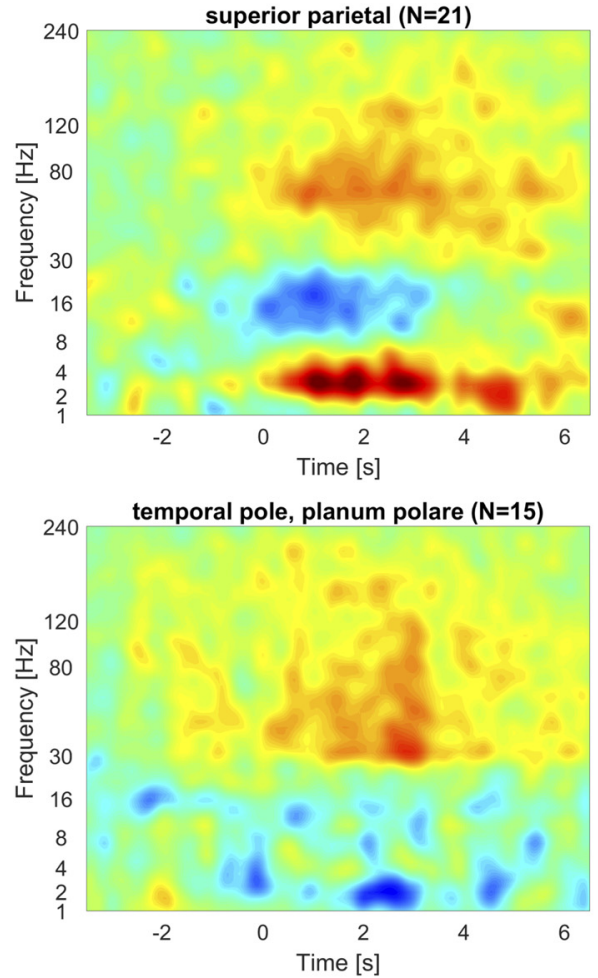
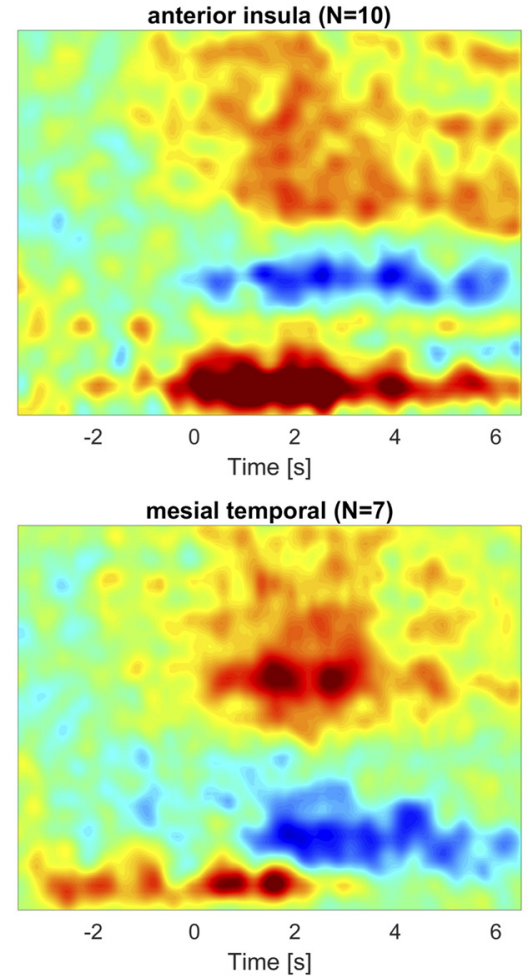
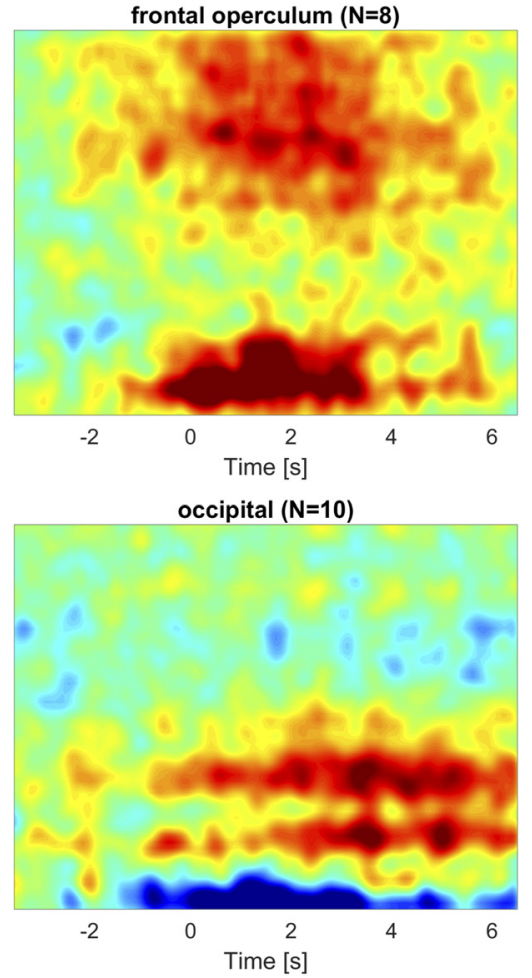

Figure 7. Time-frequency representation of SEEG signal during STW and peri-STW segments. Time zero corresponds to the onset of the STW segment. Color scale represents the difference in average power compared with the average of control segments (for all cases, the scale goes from -0.5 to $0.5 \mathrm{~dB}$ ). The number of averaged channels in each region is indicated in parentheses. In the superior parietal cortex, insula, frontal operculum, and to a less extent mesial temporal structures, an increase in both $\delta$ and $\gamma$ band activity is observed, with ripple band activity observed in the anterior insula, frontal operculum, and mesiotemporal lobe. The temporal pole and planum polare mainly present with HF activity, and the occipital cortex exhibits a $\theta$ to $\beta$ increase along with a decrease in slow waves. 
power increase between neighboring channels is correlated, but the correlation is not very high, indicating spatial variability at a small scale in the intracranial correlates of STW. Finally, the correlation between the $z$ score in the high $\delta$ and low ripple bands is far from perfect in the same channel, indicating that the variability in the channel is not limited to a stereotypical response of varying amplitude (Fig. 9).

Unsupervised clustering of all channels in three groups resulted in a group showing an increase in HF and LF, a group showing an increase in HF only, and a third group showing no change. Almost two-thirds of the channels did not change their activity during STW segments (Fig. 10A). This result, which contrasts with the above findings of a large set of regions involved, highlights the local variability in STW, within and between patients. This spatial heterogeneity is illustrated in Figure $10 B$. Some regions exhibit a prominent pattern (increase in $\mathrm{LF}$ and $\mathrm{HF}$ in the anterior insula, superior parietal cortex and supplementary motor cortex; HF increase in mesial temporal structures, posterior cingulate cortex and frontal operculum; no changes in LF and HF in the central operculum, in the superior and inferior temporal gyri as well as in the occipital cortex), but several regions include channels with variable behaviors during scalp-detected STW as found in the precuneus, the medial frontal cortex, or the middle cingulate gyrus.

\section{Discussion}

Using the unique opportunity provided by combined SEEG/PSG, we investigated local intracranial correlates of scalp-detected STW in human. We demonstrate that (1) many brain areas exhibit iSTW; (2) a very widespread increase in HF activity is observed concomitantly; and (3) iSTW present as local phenomena, with high spatiotemporal heterogeneity.

\section{iSTW involve a parieto-fronto-insular network}

We recorded iSTW in parietal lateral and medial areas, in the anterior insula, in the lateral and orbital frontal cortex, and in mesiotemporal structures. These results are consistent with the frontocentral topography of scalp-recorded STW (Yasoshima et al., 1984). Recently, an HD-EEG study identified two cortical sources for STW: a frontocentral source including the frontal superior and medial cortex and a posterior temporooccipital source (Bernardi et al., 2019). Our results partly overlap with these findings regarding frontal iSTW, but intracranial recordings, as a gold standard approach to investigate local activity, allowed us to find additional posterior parietal and insular sources, pointing toward high integration hubs. We observed $\theta$ to $\beta$ rather than $\delta$ oscillations in the occipital cortex during STW segments. This discrepancy may be explained by the fact that $70 \%$ of the analyzed occipital SEEG channels were located in the mesial part of the occipital lobe, which is not accessible to scalp EEG. It may also be related to the previous finding of a $\theta$ rather than $\delta$ peak in occipital areas during NREM sleep (von Ellenrieder et al., 2020).

\section{STW exhibit local properties}

High spatiotemporal heterogeneity was observed in the involvement of brain regions in iSTW. We also found an asynchrony in iSTW bursts between areas, which may account for the notch observed in scalp recordings resulting from locally shifted STW. Interindividual variability in STW density has previously been reported (Geisler et al., 1987; Pearl et al., 2002). This stealthy pattern of STW may explain why very few studies have been conducted to investigate their generators. Our results suggest that STW are under local cortical regulation. Accordingly, a reduction in STW has been reported after cortical lesions because of stroke (Bassetti and Aldrich, 2001).

\section{STW correspond to an activated state}

We demonstrate an increase in HF activity which may reflect a widespread cerebral activation during STW. This activation comprised the primary sensorimotor cortex, associative high order areas including major hubs of the default mode network 

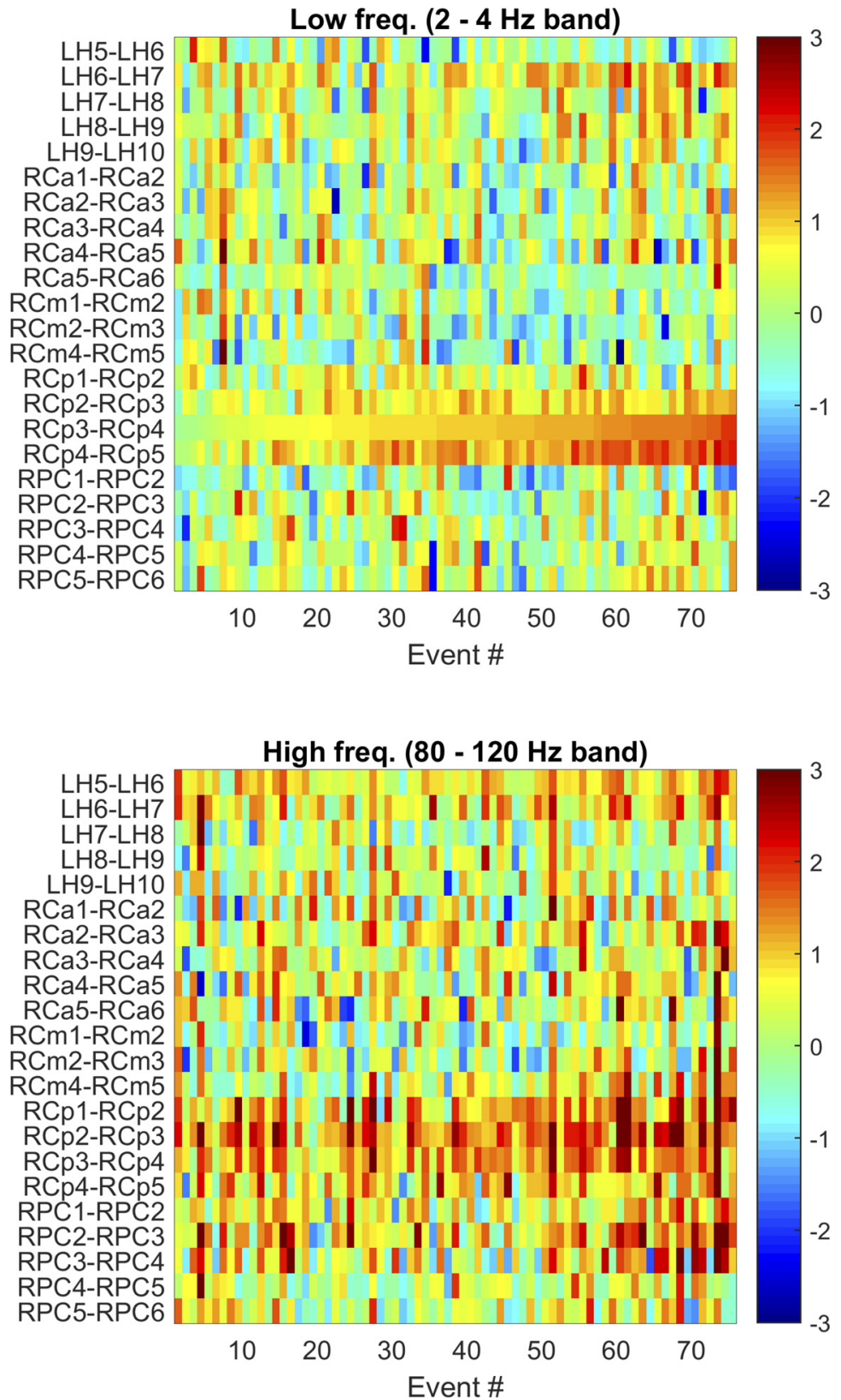

Figure 9. Spatiotemporal heterogeneity in intracranial correlates of scalp-detected STW (example Patient 18). The variation in the $z$ score with respect to the control segments in 22 channels in the high $\delta(2-4 \mathrm{~Hz}$, top) and low-ripple $(80-120 \mathrm{~Hz}$, bottom) bands during the 76 scalp STW events is presented. Segments have been sorted according to the $z$ scores of the high $\delta$ frequency band in channel $\mathrm{RCp} 3-4$, which has an important increase in both bands. In 16 of 22 channels, the effect size is larger than zero in the $2-4 \mathrm{~Hz}$ band, but only 4 channels have a large effect size (LH6-7, RCp2-3, RCp3-4, RCp4-5). The largest effect size $(d=2.06)$ is observed in channel $R C p 3-4$, and the $z$ score of the power increase for the different events in this channel shows high temporal variability as it ranges from -0.17 to 2.59 (mean \pm SD, $0.98 \pm 0.48$ ). The correlation coefficient of the time-series of the $z$ score of the power increase between channels (i.e., between rows) is not very high, indicating an important spatial variability. The neighboring channel RCp4-5 shows a correlation coefficient $(\rho=0.82)$ statistically different from zero $\left(p<10^{-4}\right.$ after correction for multiple comparisons), as do RCp2-3 $\left(\rho=0.53, p<10^{-4}\right)$ and LH6-7 $(\rho=0.44$, $p=0.005)$. In the low-ripple band $(80-120 \mathrm{~Hz})$ there are 21 of 22 channels with a positive effect size, and 8 with a large effect size (LH5-6, LH6-7, all RCp channels, and RPC1-2, RPC2-3). Finally, the behavior between the different bands is also variable; the correlation coefficient of the variability between bands $(2-4 \mathrm{~Hz}$ and $80-120 \mathrm{~Hz}$ ) is only significantly different from zero for RPC3-4 ( $\rho=0.44, p=0.005$ ). LH, Left hippocampus (deep contacts 1-5 in the hippocampus, superficial contacts from 6 to 10 in middle temporal gyrus); RCa, targeting right anterior cingulate gyrus (deep contacts 1-4 in the anterior cingulate gyrus, superficial contacts 5 and 6 in the middle frontal gyrus); $R(\mathrm{Cm}$, targeting right middle cingulate gyrus (deep contacts 1-3 in the middle cingulate gyrus, superficial contacts $4-5$ in the middle frontal gyrus); $R C p$, targeting right posterior cingulate gyrus (deep contacts 1-3 in the medial segment of precentral gyrus, superficial contacts 4 and 5 in postcentral gyrus); RPC, targeting right precuneus (deep contacts 1 and 2 in the precuneus, superficial contacts 3-5 in the superior parietal lobule, and 6 in supramarginal gyrus). Abbreviations: freq, frequencies.
(Raichle et al., 2001), and limbic areas such as cingulate cortex and mesiotemporal structures. Interestingly, this activation manifested in some regions as a decrease in $\alpha$-low $\beta$ and an increase in $\gamma$ frequencies, consistent with electrophysiological correlates of various cognitive processes during wakefulness (Pfurtscheller and Lopes da Silva, 1999; Szurhaj and Derambure, 2006; Lachaux et al., 2012). $\mu$ desynchronization and $\gamma$ synchronization have been previously reported in the motor cortex during phasic compared with tonic REM sleep using SEEG; this cortical activation during phasic REM sleep was similar to that observed during wakefulness voluntary movements (De Carli et al., 2016). In our work, we extend this finding to other brain areas and highlight that such activations occur at the time of STW, regardless of the ongoing tonic or phasic REM sleep state.

\section{Phasic versus tonic REM sleep}

Our observation of different REM sleep microstates associated with various levels of cortical activation echoes previous reports about REM sleep heterogeneity, usually presented as a dichotomy between phasic and tonic REM sleep (Simor et al., 2020). The lower arousal thresholds and the higher $\gamma$ activity in phasic versus tonic REM sleep have led to the concept that phasic REM sleep represents a more "internally focused" but cognitively active state, which could explain the occurrence of dreams (Gross and Gotman, 1999; Wehrle et al., 2007; Ermis et al., 2010; Simor et al., 2016). Recently, using HDEEG, Simor et al. (2019) identified two clusters for HF activity in phasic REM sleep: one involving most parts of the frontal lobe and the other the inferolateral temporal, occipital, and medial parietal cortices (Simor et al., 2019). Most of these regions overlap with our results regarding $\gamma$ activities, except for the occipital cortex. However, our observations were not based on the comparison between phasic and tonic REM sleep, which points to the fact that phasic REM sleep does not totally coincide with REM sleep with bursts of REM. This is in line with several works about dissociated behaviors of the various components of phasic REM sleep in experimental or pathologic conditions (Geisler et al., 1987; Pinto et al., 2002). Nevertheless, given the particular temporal relationship between STW and bursts of REM (Sato et al., 1997), confirmed in our study, it may be hypothesized that STW represent the border between tonic and phasic REM sleep.

\section{Functional significance of STW cortical activations}

We observed a strong increase in ripple rates in regions involved in iSTW and in other areas, such as the hippocampus. Like NREM slow waves synchronizing spindles and ripples, STW may drive faster activities (Clemens et al., 2007; Bernardi et al., 2019). Given the role of ripples 
in replay phenomena, such STW-driven activations may participate in REM sleep memory functions (Louie and Wilson, 2001; Ji and Wilson, 2007). The "patchy" nature of iSTW, with various involvement of cortical regions from one STW to another, may orchestrate synchronized reactivations in different networks, allowing strengthening or tagging of complex representations which have been reorganized during NREM sleep (Rasch and Born, 2013). This process may target more specifically emotionally salient information and lead to their contextualization (Walker and van der Helm, 2009) as suggested by our results showing activation of limbic but also several associative cortices and the insula. Mental contents evoked during these short strongly activated periods could participate to the emergence of dreams as epiphenomena.

\section{STW may be the cortical component of} ponto-geniculo-cortical transients

Our finding of a mesio-occipital $\theta$ activity during STW segments may correspond to the previously reported $\theta$ waves in the human visual cortex during phasic REM sleep, considered as the occipital part of PGO waves (Frauscher et al., 2018a). The relationship between STW and PGO waves remains a matter of debate. PGO waves have been initially described in cats as bursts of sharp waves time-locked with REM (Michel et al., 1964). They are generated in the pontine tegmentum and propagate to the thalamic lateral geniculate nuclei and to the occipital cortex (Callaway et al., 1987; Datta, 1997). They have been postulated to inform the visual system about eye movements and to promote REM sleep-dependent neuronal plasticity (Callaway et al., 1987; Datta et al., 2004). However, PGO waves are not confined to the visual cortex but rather spread to different brain areas where they may modulate neuronal firing and synchronization of fast oscillations (Amzica and Steriade, 1996; Andrillon et al., 2015). A link between PGO and STW has been proposed by Ishiguro et al. (1979) who found a temporal association between STW and bursts of REM (Ishiguro et al., 1979). The only recordings of STW in animals were performed in nonhuman primates (Snyder et al., 1978; Balzamo, 1980). These widespread ponto-geniculo-cortical waves, interpreted as being the counterpart of PGO described in cats, were variable in location from one burst to another and exhibited a morphology close to that of STW (Balzamo, 1980). One could hypothesize that STW, like PGO waves, may be associated with cortical activations initiated by phasic ascending brainstem inputs and be linked to motor outputs such as bursts of REM. This is in keeping with alterations of STW in neurologic diseases affecting the brainstem, such as bulbar forms of the postpolio syndrome where STW appear delayed (H. Siegel et al., 1999).

A

B
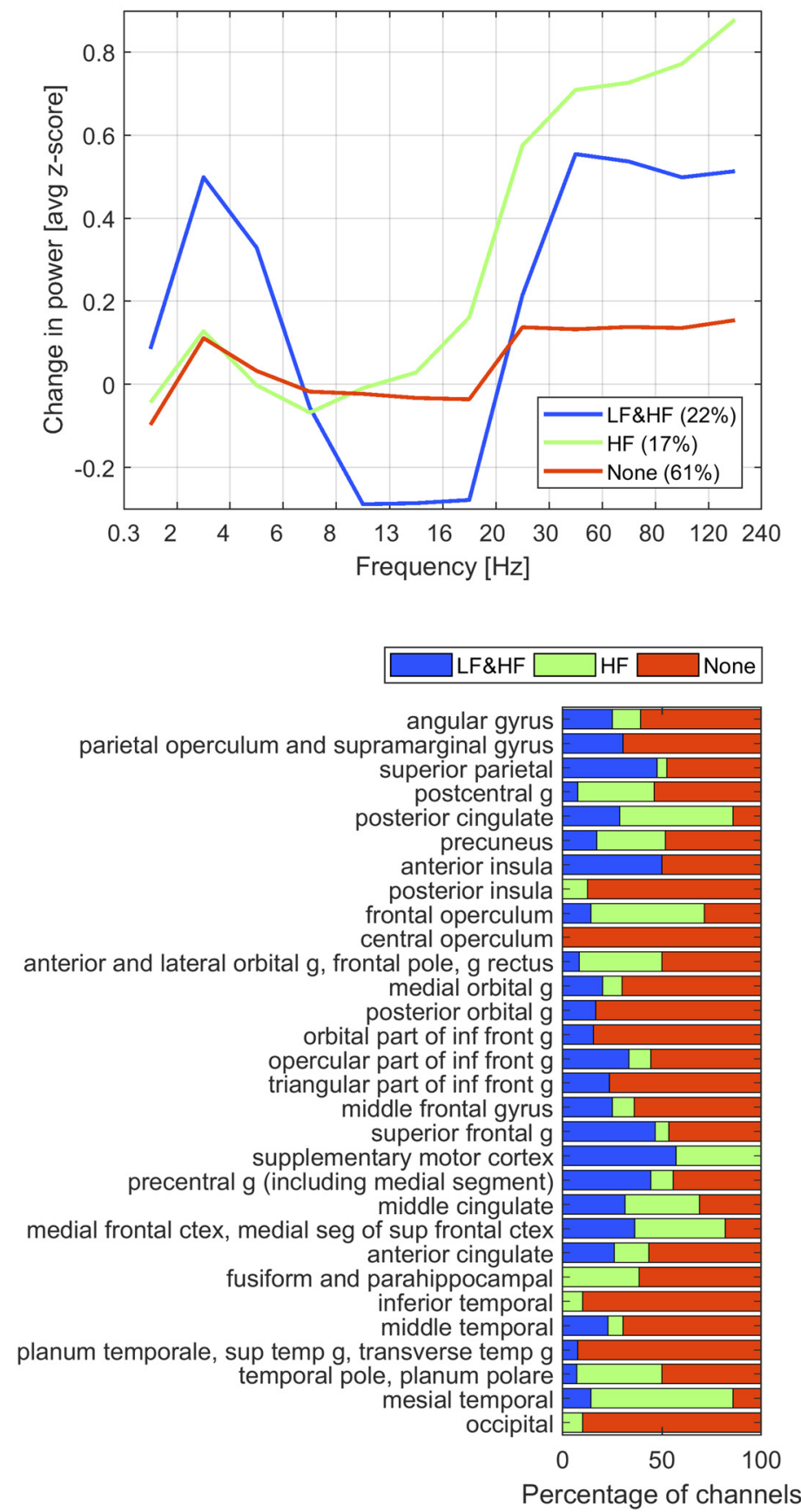

Figure 10. Typical behaviors of SEEG correlates of STW. A, Clustering of SEEG channel according to HF and LF changes during STW. The figure shows the typical behavior of three automatically determined groups of channels. The grouping was done in 514 of the 544 channels, after excluding channels with outliers in one or more of the bands that would render the clustering algorithm inefficient. The choice of three groups was arbitrary. The main behaviors were an increase in the $z$ score in LF and $\mathrm{HF}$, an increase in $\mathrm{HF}$ only, and no change. In most channels $(61 \%)$, the correlate to scalp STW is weak. These three groups of channels are usually present in all brain regions, and the difference in the average correlate in each region is to some degree because of the difference in the proportion of these three groups of channels. B. Spatial heterogeneity in SEEG correlates of STW. The distribution of channels showing the three previously determined pattern (LF and $\mathrm{HF}, \mathrm{HF}$, and none) in each brain region is presented as a percentage. In most brain areas, channel involvement in LF and HF is not homogeneous. Abbreviations: $g$, gyrus.

\section{Strengths and potential limitations}

We performed the first intracranial study of STW using a large dataset of combined SEEG/PSG recordings. The independent double visual marking of STW allowed a specific detection of events. Using a wide frequency band analysis of intracranial 
EEG, we were able to identify STW-related changes in LF and HF activity without an a priori hypothesis. We have to acknowledge that data were obtained in epilepsy patients, as epilepsy is the only condition where SEEG is performed in the human. However, modifications of STW reported in epilepsy concern density, duration, and frequency, which were not the focus of our work (Vega-Bermudez et al., 2005). It is unlikely that antiepileptic drugs may have modified STW, as most of them do not affect REM sleep (Jain and Glauser, 2014) and the scalp-EEG characteristics of our selected STW were similar to those observed in healthy subjects (Sato et al., 1997). Spatial sampling may be considered as heterogeneous, which is a limitation inherent to SEEG. We overcame this potential bias by including a large number of patients whose results were superimposed on one brain after standardization of intracranial electrodes positions in a common 3D environment.

In conclusion, STW are fronto-parieto-insular oscillations exhibiting a high spatiotemporal heterogeneity, suggesting local cortical regulation. They are associated with widespread brain activation during the tonic to phasic REM sleep transition and may represent a particular time window for multifocal replay phenomena. Their relationship with subcortical activations and their functional significance, especially regarding REM sleep cognitive functions, remain to be investigated.

\section{References}

Amzica F, Steriade M (1996) Progressive cortical synchronization of ponto-geniculo-occipital potentials during rapid eye movement sleep. Neuroscience 72:309-314.

Andrillon T, Nir Y, Cirelli C, Tononi G, Fried I (2015) Single-neuron activity and eye movements during human REM sleep and awake vision. Nat Commun 6:7884.

Balzamo E (1980) States of wakefulness and ponto-geniculo-cortical activities (PGC) in Papio anubis (author's transl). Electroencephalogr Clin Neurophysiol 48:694-705.

Bassetti CL, Aldrich MS (2001) Sleep electroencephalogram changes in acute hemispheric stroke. Sleep Med 2:185-194.

Berger R, Olley P, Oswald I (1962) The EEG, eye movements and dreams of the blind. Q J Exp Psychol 14:183-186.

Bernardi G, Betta M, Ricciardi E, Pietrini P, Tononi G, Siclari F (2019) Regional delta waves in human rapid-eye movement sleep. J Neurosci 39:2686-2697.

Berry RB, Brooks R, Gamaldo CE, Harding SM, Lloyd R, Marcus CL, Vaughn BV (2017) The AASM manual for the scoring of sleep and associated events: rules, terminology and technical specifications, Version 2.4. Darien, IL: American Academy of Sleep Medicine.

Boyce R, Williams S, Adamantidis A (2017) REM sleep and memory. Curr Opin Neurobiol 44:167-177.

Broughton R, Hasan J (1995) Quantitative topographic electroencephalographic mapping during drowsiness and sleep onset. J Clin Neurophysiol 12:372-386.

Callaway CW, Lydic R, Baghdoyan HA, Hobson JA (1987) Pontogeniculooccipital waves: spontaneous visual system activity during rapid eye movement sleep. Cell Mol Neurobiol 7:105-149.

Campana C, Zubler F, Gibbs S, de Carli F, Proserpio P, Rubino A, Cossu M, Tassi L, Schindler K, Nobili L (2017) Suppression of interictal spikes during phasic rapid eye movement sleep: a quantitative stereo-electroencephalography study. J Sleep Res 26:606-613.

Carskadon MA, Dement WC (2017) Normal human sleep: an overview. In: Principles and practice of sleep medicine, Ed 6 (Kryger MH, Dement WC, eds), pp 15-24. Philadelphia: Elsevier.

Clemens Z, Molle M, Eross L, Barsi P, Halasz P, Born J (2007) Temporal coupling of parahippocampal ripples, sleep spindles and slow oscillations in humans. Brain 130:2868-2878.

Clemens Z, Weiss B, Szucs A, Eross L, Rasonyi G, Halasz P (2009) Phase coupling between rhythmic slow activity and gamma characterizes mesiotemporal rapid-eye-movement sleep in humans. Neuroscience 163:388-396.
Datta S (1997) Cellular basis of pontine ponto-geniculo-occipital wave generation and modulation. Cell Mol Neurobiol 17:341-365.

Datta S, Mavanji V, Ulloor J, Patterson EH (2004) Activation of phasic pontine-wave generator prevents rapid eye movement sleep deprivationinduced learning impairment in the rat: a mechanism for sleep-dependent plasticity. J Neurosci 24:1416-1427.

De Carli F, Proserpio P, Morrone E, Sartori I, Ferrara M, Gibbs SA, De Gennaro L, Lo Russo G, Nobili L (2016) Activation of the motor cortex during phasic rapid eye movement sleep. Ann Neurol 79:326-330.

Dement W, Kleitman N (1957) Cyclic variations in EEG during sleep and their relation to eye movements, body motility, and dreaming. Electroencephalogr Clin Neurophysiol 9:673-690.

Diekelmann S, Born J (2010) The memory function of sleep. Nat Rev Neurosci 11:114-126.

Drouin S, Kochanowska A, Kersten-Oertel M, Gerard IJ, Zelmann R, De Nigris D, Beriault S, Arbel T, Sirhan D, Sadikot AF, Hall JA, Sinclair DS, Petrecca K, DelMaestro RF, Collins DL (2017) IBIS: an OR ready opensource platform for image-guided neurosurgery. Int J Comput Assist Radiol Surg 12:363-378.

Ermis U, Krakow K, Voss U (2010) Arousal thresholds during human tonic and phasic REM sleep. J Sleep Res 19:400-406.

Frauscher B, von Ellenrieder N, Dubeau F, Gotman J (2015) Scalp spindles are associated with widespread intracranial activity with unexpectedly low synchrony. Neuroimage 105:1-12.

Frauscher B, Joshi S, von Ellenrieder N, Nguyen DK, Dubeau F, Gotman J (2018a) Sharply contoured theta waves are the human correlate of pontogeniculo-occipital waves in the primary visual cortex. Clin Neurophysiol 129:1526-1533.

Frauscher B, von Ellenrieder N, Zelmann R, Rogers C, Nguyen DK, Kahane P, Dubeau F, Gotman J (2018b) High-frequency oscillations in the normal human brain. Ann Neurol 84:374-385.

Frauscher B, von Ellenrieder N, Zelmann R, Dolezalova I, Minotti L, Olivier A, Hall J, Hoffmann D, Nguyen DK, Kahane P, Dubeau F, Gotman J (2018c) Atlas of the normal intracranial electroencephalogram: neurophysiological awake activity in different cortical areas. Brain 141:11301144.

Geisler P, Meier-Ewert K, Matsubayshi K (1987) Rapid eye movements, muscle twitches and sawtooth waves in the sleep of narcoleptic patients and controls. Electroencephalogr Clin Neurophysiol 67:499-507.

Gross DW, Gotman J (1999) Correlation of high-frequency oscillations with the sleep-wake cycle and cognitive activity in humans. Neuroscience 94:1005-1018.

Ishiguro T, Hanamura S, Okata T (1979) The saw-tooth wave associated with small nystagmus: a study on a narcoleptic patient and her family. Sleep Res 8:195.

Ives JR (2005) New chronic EEG electrode for critical/intensive care unit monitoring. J Clin Neurophysiol 22:119-123.

Jain A, Dubes R (1988) Algorithms for clustering data. Upper Saddle River, NJ: Prentice-Hall.

Jain SV, Glauser TA (2014) Effects of epilepsy treatments on sleep architecture and daytime sleepiness: an evidence-based review of objective sleep metrics. Epilepsia 55:26-37.

Ji D, Wilson MA (2007) Coordinated memory replay in the visual cortex and hippocampus during sleep. Nat Neurosci 10:100-107.

Jiang X, Gonzalez-Martinez J, Halgren E (2019) Coordination of human hippocampal sharpwave ripples during NREM sleep with cortical theta bursts, spindles, downstates, and upstates. J Neurosci 39:8744-8761.

Jouvet M, Michel F (1959) [Electromyographic correlations of sleep in the chronic decorticate and mesencephalic cat]. C R Seances Soc Biol Fil 153:422-425.

Jouvet M, Michel F, Mounier D (1960) [Comparative electroencephalographic analysis of physiological sleep in the cat and in man]. Rev Neurol (Paris) 103:189-205.

Lachaux JP, Axmacher N, Mormann F, Halgren E, Crone NE (2012) Highfrequency neural activity and human cognition: past, present and possible future of intracranial EEG research. Prog Neurobiol 98:279-301.

Landman B, Warfield S (2012) 2012 workshop on multi-atlas labeling. Create Space Indendant Publushing Plateform. Scotts Valley, CA.

Latreille V, von Ellenrieder N, Peter-Derex L, Dubeau F, Gotman J, Frauscher B (2020) The human K-complex: insights from combined scalp-intracranial EEG recordings. Neuroimage 213:116748. 
Lim AS, Lozano AM, Moro E, Hamani C, Hutchison WD, Dostrovsky JO, Lang AE, Wennberg RA, Murray BJ (2007) Characterization of REMsleep associated ponto-geniculo-occipital waves in the human pons. Sleep 30:823-827.

Louie K, Wilson MA (2001) Temporally structured replay of awake hippocampal ensemble activity during rapid eye movement sleep. Neuron 29:145-156.

Luppi PH, Fort P (2019) Sleep-wake physiology. Handb Clin Neurol 160:359-370.

Michel F, Jeannerod M, Mouret J, Rechtschaffen A, Jouvet M (1964) [On the mechanisms of activity of points at the level of the visual system during the paradoxal phase of sleep]. C R Seances Soc Biol Fil 158:103-106.

Montgomery SM, Sirota A, Buzsaki G (2008) Theta and gamma coordination of hippocampal networks during waking and rapid eye movement sleep. J Neurosci 28:6731-6741.

Nelson JP, McCarley RW, Hobson JA (1983) REM sleep burst neurons, PGO waves, and eye movement information. J Neurophysiol 50:784-797.

Nir Y, Staba RJ, Andrillon T, Vyazovskiy VV, Cirelli C, Fried I, Tononi G (2011) Regional slow waves and spindles in human sleep. Neuron 70:153-169.

Pearl PL, LaFleur BJ, Reigle SC, Rich AS, Freeman AA, McCutchen C, Sato S (2002) Sawtooth wave density analysis during REM sleep in normal volunteers. Sleep Med 3:255-258.

Peigneux P, Laureys S, Fuchs S, Delbeuck X, Degueldre C, Aerts J, Delfiore G, Luxen A, Maquet P (2001) Generation of rapid eye movements during paradoxical sleep in humans. Neuroimage 14:701-708.

Peter-Derex L, Comte JC, Mauguière F, Salin PA (2012) Density and frequency caudo-rostral gradients of sleep spindles recorded in the human cortex. Sleep 35:69-79.

Pfurtscheller G, Lopes da Silva FH (1999) Event-related EEG/MEG synchronization and desynchronization: basic principles. Clin Neurophysiol 110:1842-1857.

Pinto LR Jr, Peres CA, Russo RH, Remesar-Lopez AJ, Tufik S (2002) Sawtooth waves during REM sleep after administration of haloperidol combined with total sleep deprivation in healthy young subjects. Braz J Med Biol Res 35:599-604.

Raichle ME, MacLeod AM, Snyder AZ, Powers WJ, Gusnard DA, Shulman GL (2001) A default mode of brain function. Proc Natl Acad Sci USA 98:676-682.

Rasch B, Born J (2013) About sleep's role in memory. Physiol Rev 93:681766.

Rechtschaffen A, Kales A (1968) A manual of standardized terminology, techniques and scoring system for sleep stage of human subjects. Los Angeles: Brain Information Service/Brain Research Institute, University of California.

Rodenbeck A, Binder R, Geisler P, Danker-Hopfe H, Lund R, Raschke F, Weeß HG, Schulz H (2006) A review of sleep EEG patterns: I. A compilation of amended rules for their visual recognition according to Rechtschaffen and Kales. Somnologie 10:159-175.

Sato S, McCutchen C, Graham B, Freeman A, von Albertini-Carletti I, Alling DW (1997) Relationship between muscle tone changes, sawtooth waves and rapid eye movements during sleep. Electroencephalogr Clin Neurophysiol 103:627-632.

Siegel H, McCutchen C, Dalakas MC, Freeman A, Graham B, Alling D, Sato S (1999) Physiologic events initiating REM sleep in patients with the postpolio syndrome. Neurology 52:516-522.

Siegel J (2011) REM sleep. In: Principles and practice of sleep medicine, Ed 5 (Kryger MH, Dement WC, eds, pp 92-111. St. Louis: Elsevier Saunders.

Simor P, Gombos F, Szakadat S, Sandor P, Bodizs R (2016) EEG spectral power in phasic and tonic REM sleep: different patterns in young adults and children. J Sleep Res 25:269-277.

Simor P, van Der Wijk G, Gombos F, Kovács I (2019) The paradox of rapid eye movement sleep in the light of oscillatory activity and cortical synchronization during phasic and tonic microstates. Neuroimage 202:116066.

Simor P, van der Wijk G, Nobili L, Peigneux P (2020) The microstructure of REM sleep: why phasic and tonic? Sleep Med Rev 52:101305.

Snyder EW, Dustman RE, Johnson RL (1978) Sawtooth waves: concomitants of rapid eye movement sleep in monkeys. Electroencephalogr Clin Neurophysiol 45:111-113.

Staresina BP, Bergmann TO, Bonnefond M, van der Meij R, Jensen O, Deuker L, Elger CE, Axmacher N, Fell J (2015) Hierarchical nesting of slow oscillations, spindles and ripples in the human hippocampus during sleep. Nat Neurosci 18:1679-1686.

Steriade M (2003) The corticothalamic system in sleep. Front Biosci 8:d878d899.

Steriade M, McCormick DA, Sejnowski TJ (1993) Thalamocortical oscillations in the sleeping and aroused brain. Science 262:679-685.

Szurhaj W, Derambure P (2006) Intracerebral study of gamma oscillations in the human sensorimotor cortex. Prog Brain Res 159:297-310.

Vega-Bermudez F, Szczepanski S, Malow B, Sato S (2005) Sawtooth wave density analysis during REM sleep in temporal lobe epilepsy patients. Sleep Med 6:367-370.

von Ellenrieder N, Gotman J, Zelmann R, Rogers C, Nguyen DK, Kahane P, Dubeau F, Frauscher B (2020) How the human brain sleeps: direct cortical recordings of normal brain activity. Ann Neurol 87:289-301.

Walker MP, van der Helm E (2009) Overnight therapy? The role of sleep in emotional brain processing. Psychol Bull 135:731-748.

Wehrle R, Kaufmann C, Wetter TC, Holsboer F, Auer DP, Pollmacher T, Czisch M (2007) Functional microstates within human REM sleep: first evidence from fMRI of a thalamocortical network specific for phasic REM periods. Eur J Neurosci 25:863-871.

Yasoshima A, Hayashi H, Iijima S, Sugita Y, Teshima Y, Shimizu T, Hishikawa Y (1984) Potential distribution of vertex sharp wave and saw-toothed wave on the scalp. Electroencephalogr Clin Neurophysiol 58:73-76. 